\title{
All-Quad Meshing without Cleanup
}

\author{
Ahmad A. Rushdi ${ }^{\mathrm{a}, \mathrm{b}, *}$, Scott A. Mitchell ${ }^{\mathrm{b}}$, Ahmed H. Mahmoud ${ }^{\mathrm{c}}$, Chandrajit C. Bajaj ${ }^{\mathrm{a}}$, Mohamed S. Ebeida \\ ${ }^{a}$ Institute for Computational Engineering and Sciences, University of Texas, Austin, TX, USA \\ ${ }^{b}$ Sandia National Laboratories, Albuquerque, NM, USA \\ ${ }^{c}$ University of California, Davis, CA, USA
}

\begin{abstract}
We present an all-quad meshing algorithm for general domains. We start with a strongly balanced quadtree. In contrast to snapping the quadtree corners onto the geometric domain boundaries, we move them away from the geometry. Then we intersect the moved grid with the geometry. The resulting polygons are converted into quads with midpoint subdivision. Moving away avoids creating any flat angles, either at a quadtree corner or at a geometryquadtree intersection. We are able to handle two-sided domains, and more complex topologies than prior methods. The algorithm is provably correct and robust in practice. It is cleanup-free, meaning we have angle and edge length bounds without the use of any pillowing, swapping, or smoothing. Thus, our simple algorithm is fast and predictable. This paper has better quality bounds, and the algorithm is demonstrated over more complex domains, than our prior version.
\end{abstract}

Keywords: All-Quadrilateral Meshing, Guaranteed Quality, Sharp Features

\section{Introduction}

Generating good quality meshes is a challenging problem with many engineering applications: e.g., Finite Element Analysis (FEA) [1, 2, 3] and ComputerAided Design (CAD) $[4,5]$. The quality of the mesh plays a significant role in the accuracy and stability of the numerical computation. It is difficult to determine if a mesh possesses even the minimal quality necessary to undertake a computational analysis without concrete metrics. Since the necessary criteria are often application specific, the community has gravitated toward quality metrics based on geometric criteria that are usually sufficient. For example, angle minimum and maximum bounds are commonly used, along with element size, aspect ratio, skew, stretching, and orientation.

\footnotetext{
This paper is extended from an earlier version published in the proceedings of the International Meshing Roundtable (IMR) 2015.

This work was sponsored by the Laboratory Directed Research and Development (LDRD) Program at Sandia National Laboratories. Sandia National Laboratories is a multi-program laboratory managed and operated by Sandia Corporation, a wholly owned subsidiary of Lockheed Martin Corporation, for the U.S. Department of Energy's National Nuclear Security Administration under contract DE-AC0494AL85000.

${ }^{*}$ Corresponding author

Email address: arushdi@utexas .edu (Ahmad A. Rushdi)
}

Preprint submitted to Journal of Computer-Aided Design

\subsection{Related Work}

Unstructured all-quad meshing algorithms are usually categorized into two main categories: indirect and direct. A classical indirect approach starts with a triangular mesh, and then transforms the triangular elements into quadrilateral elements, via optimization $[6,7]$, refinement and coarsening [8], or simplification [9]. A class of indirect methods start with a triangular mesh and applies the mid-point subdivision rule $[10,11]$ to

May 24, 2016 
split a triangle into three quad elements. This can be 93 generalized to other local subdivision operations. For example, diamond-kite [12] applies recursive subdivision based on a regular tiling composed of only diamonds and kites, but does not handle domain boundaries. Q-Morph [13] is a popular indirect approach that follows a sequence of systematic triangle transformations to create an all-quadrilateral mesh. However, Q-Morph requires topological cleanup and smoothing to guarantee the quality of the final all-quad mesh. Q-Tran [14] is another indirect algorithm that produces quadrilaterals with provably-good quality without a smoothing post-processing step, and manages to handle domain boundaries. Nevertheless, the class of indirect methods typically suffers from a large number of irregular nodes that are connected to more (or less) than four mesh elements, which is typically undesired in numerical simulations.

Direct approaches construct quadrilaterals without an intermediate triangular mesh. The advancing front algorithms (e.g., paving [15]) generate all quad meshes by placing mesh points on the boundaries of the input domain, then recursively projecting edges on the front towards the interior of the domain to form quads [16, 17]. However, these typically suffer from stability problems that require heuristic cleanup operations. Grid based methods construct a uniform Cartesian or quadtree background grid then modify it to conform to the domain boundaries [18, 19]. These methods are easy to implement. They can often provide quality guarantees [20, 21, 22]. However, many variations produce flat or inverted elements before cleanup and smoothing, by snapping multiple grid points to lie exactly along some curve. Square packing [23] and circle packing [24] methods can generate all quads. Circle packing bounds the maximum angle to $120^{\circ}$, but does not bound the minimum angle. In Ataly et al. [25], the minimum angle is bounded.

Other methods include surface parameterization and ${ }_{109}$ orientation field methods [26, 27, 28] which find the 110 smoothest orientation field on a surface subject to a 111 target direction or boundary conditions. Similarly, the 112 Quadcover [29] approach computes a global continu- ${ }_{113}$ ous parameterization for an arbitrary given simplicial ${ }_{114}$ 2-manifold. However, it is not trivial to find globally 115 optimal orientation solutions.

In this paper, we describe a direct quadtree-based al- 117 gorithm that produces an all-quad mesh. It is stable, and ${ }_{118}$ all operations are simple and local. The output mesh 119 conforms to both the interior and exterior of the do- 120 main geometry. It has provably-good quality without 121 post-processing cleanup. The key innovation is to move 122 grid points away from the geometry, rather than snapping onto the geometry. An earlier version of this paper was published in the proceedings of the International Meshing Roundtable (IMR) 2015 [30]. Here we have dramatically improved the provable quality bounds, and demonstrated the method on more complex domains.

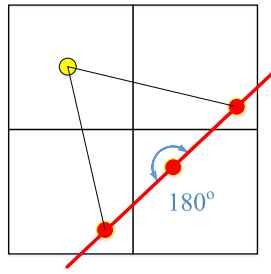

(a) Dual contouring.

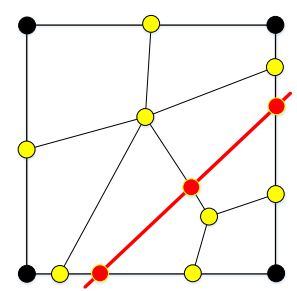

(b) Our algorithm
Figure 1: Starting with a uniform grid (black): comparing mesh element quality of our algorithm to that of dual contouring when an element is intersected by a domain boundary (red). Dual contouring generates a dual quad element whose vertices are preferably on the boundary (red) or the center of a non-intersected element (yellow), which often results in an undesired flat angle degrading the mesh quality. Our algorithm modifies the existing grid by adding points at the boundary intersections (red), splitting the intersected element, adding refinement points (yellow) at the middle of all edges and centers of split elements, and refining the split elements into well-shaped allquad elements with good angles bounds.

\subsection{Comparison to Dual Contouring}

Dual contouring is a direct all quad meshing technique that has attracted recent attention [31, 32, 33]. It starts with a uniform structured quadrilateral mesh. It is intersected with the domain geometry to produce polygons, which are then dualized to form an all-quad mesh. We highlight a few key advantages for our algorithm over dual contouring:

- Element Quality: Angle Bounds. Dual contouring adds points exactly on the domain's boundary, creating a large number of flat or close to flat angles which degrades the quality of the resulting mesh. To remove the flat angles, dual contouring requires post-processing "pillowing” [34]: a layer of quads is added between the geometry and the flat angle. Pillowing gives smoothing the freedom to move the node of the flat angle. In contrast, our algorithm creates a grid with points that are far from the boundary. Intersecting the grid with the boundary creates elements with balanced angles and aspect ratio. Our method is clean-up free, and avoids pillowing and smoothing. Figure 1 contrasts the flat angles from dual contouring with our balanced angles. 
- Employing Quadtrees. A desired mesh feature ${ }_{169}$ is to allocate more points around the boundary 170 and less far from it. Our algorithm can achieve 171 that since it starts with a balanced quadtree refine- 172 ment, positioning more points "around" the do- 173 main's boundary. Dual contouring requires a uni- 174 form grid, and using a quadtree would create nonquad elements.

- Local versus Global Operations. Modifying an existing dual contouring mesh requires the creation of the dual mesh, and pillowing operations propagate. Our algorithm, on the other hand, can easily adapt to any input domain modification by applying the same steps locally in the neighborhood of the domain modification.

\subsection{Terminology}

To easily distinguish between the initial grid, the domain geometry, and the final mesh, we use the following terminology. The initial quadtree grid is composed of squares, each of which has four sides and corners. Corners of adjacent smaller squares appear as hanging nodes. The side length of a square is denoted $s$. (A uniform Cartesian grid is a special case of a quadtree.) The geometric domain is composed of curves (which are straight line segments, and two-sided) and vertices. The intersection of the ${ }^{194}$ quadtree and geometry results in polygons and corner 195 points connected by segments. Polygons are meshed with midpoint subdivision; a midpoint subdivides each segment, and a center is placed interior to the polygon and connected to each midpoint. The final all-quad mesh is composed of elements, edges, and nodes.

\section{Algorithm}

Our algorithm is described as a set of repelling, split- ${ }^{204}$ ting, and refinement steps. These are illustrated in Fig- 205 ure 2 for the simple case of a geometric circle and uni- 206 form grid. We summarize these steps then explain the ${ }^{207}$ details in the following subsections.

1. Start with a strongly-balanced quadtree, refined to ${ }^{200}$ the level that vertices and disjoint curves do not appear in the same square.

2. Perturb the quadtree by repelling mesh points away ${ }_{213}$ from the geometry. All squares intersected by a 214 vertex or curve are split into polygons, which con- 215 form to the geometry.
3. Apply the midpoint subdivision rule to split polygons into quad elements. Some other elements will possess hanging nodes.

4. Employ the two-refinement templates to resolve all hanging nodes, generating the final conforming allquad mesh with good quality.

\subsection{Quadtree Initialization, Refinement, and Balanc- ing.}

We start with a Cartesian grid and refine it to form a quadtree that captures the fine details of the geometric domain boundaries. In principle one could start with another decomposition. Our algorithm requires that the quadtree is strongly balanced, where corner-adjacent squares differ in size by at most one; see Figure 3 for an illustration. For simplicity, in this version we chose uniform square sizes along the boundaries.

\subsection{Perturbing the Quadtree}

The goal of this step is to prevent small mesh edges and flat and sharp angles. A raw geometry-quadtree intersection might be arbitrarily close to another point, or at a very small angle. We repel corners of squares away from nearby geometry, to be at least distance $\delta$ from geometry. We choose $\delta$ to be $\frac{s}{4}$, where $s$ is the size of the square containing the corner. We have two repelling strategies, as shown in Figure 4

- Geometry-Normal Repelling. Corners are moved normal to a curve until they are $\delta$ from it.

- Grid-Aligned Repelling. Corners are moved horizontally or vertically until they are $\delta$ from a curve in the horizontal or vertical direction. A horizontal curve has absolute slope less than one; otherwise it is vertical. We move a corner vertically away from a horizontal curve, and vice versa.

\subsection{Polygon Formation}

Squares cut by the geometry are split into polygons. Squares cut by a single curve are split into two polygons, either a triangle and a pentagon, or two quadrilaterals. To recover a conforming all-quad mesh, we refine these polygons using the midpoint subdivision rule. Figure 5 shows example elements before and after refinement. We refine squares cut by two or more curves until only one curve cuts a square. Note that when two curves meet at a common vertex at a sharp angle, the element containing that angle will have poor quality. In this case we do not refine adjacent squares to separate these curves. The mesh between the two curves may have stretched elements of poor quality, and the $\delta$ separation may not be achievable. 


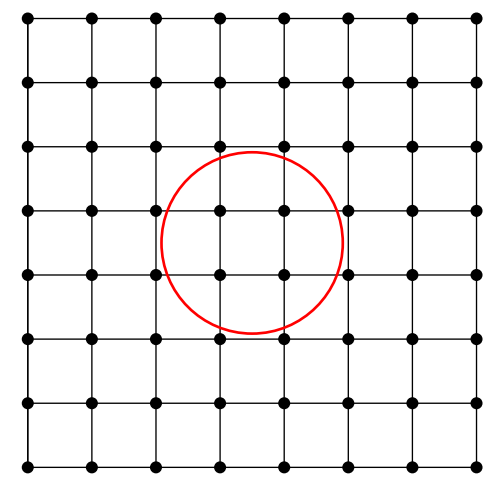

(a) Initial uniform grid (black) and a circleshaped input geometry (red).

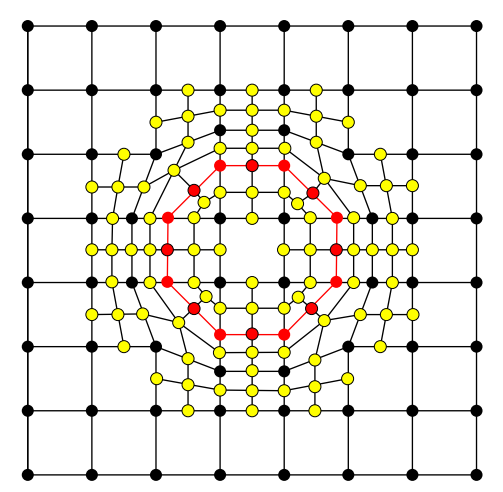

(c) Intersected and deformed elements refined into all quadrilateral elements.

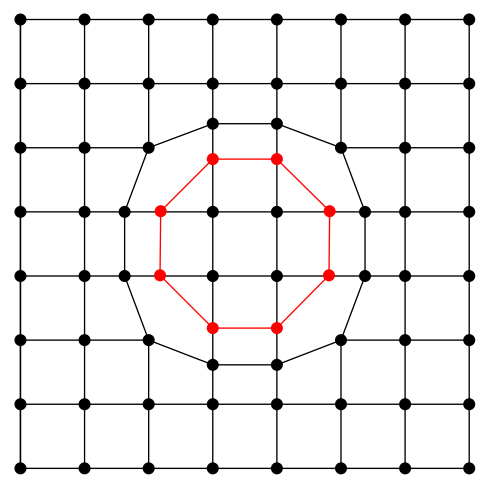

(b) Grid nodes repelled away, and mesh elements split by input boundary.

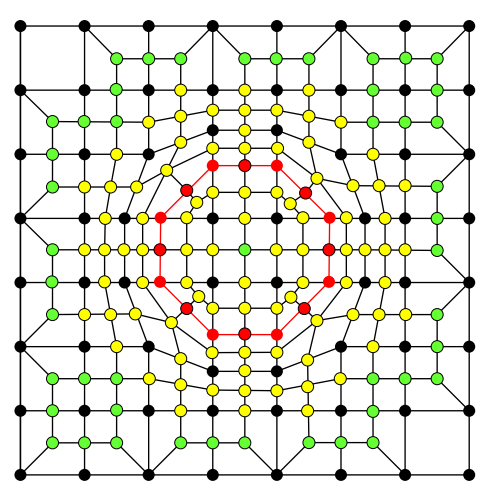

(d) 2-ref template applied to get rid of any hanging nodes in the final all-quad mesh.

Figure 2: An overview of the all-quad meshing algorithm, applied to a circle shape. Starting with a uniform Cartesian grid, we repel points that are too close to the boundary away from it with a ratio of the initial grid spacing, and split all elements that are intersected by the boundary. Each split or deformed element is then refined into four or more all-quad elements using the mid-point subdivision rule. Finally, we apply the two-refinement templates to guarantee conforming refinement with no hanging nodes.

\subsubsection{Handling Geometric Vertices}

We seek to preserve sharp features of the input ${ }^{236}$ boundary domain without introducing new ones. We ${ }^{237}$ require that a square contains at most one vertex. We ${ }^{23}$ insert a new mesh point coincident with a domain vertex, and split any element that contains a vertex before we apply midpoint subdivision.

Figure 6 shows examples of initial, split, and refined elements for a vertex. We consider isolated vertices, as well as vertices with one or more curves. In principle a vertex may have an arbitrary number of curves. For each square side that does not intersect a curve, we introduce its midpoint as a mesh point, and pull it closer to the sharp corner (a distance of $s / 8$, where $s$ is the square side length) to avoid creating flat angles in the adjacent elements. Squares are split into multiple re- 249 gions by the curves, and vertex-midpoint edges. Each 250 region is a polygon that is meshed with midpoint subdi- 251 vision independently. Adjacent squares are also meshed with midpoint subdivision, introducing hanging nodes on some of their neighbors. These, along with hanging nodes from adjacent squares being refined to a different level, are resolved in the last step of the algorithm.

Note that extreme sharp corner case might result in trapped grid points with no enough space for repelling. In this case, we position the grid point half-way between the trapping geometry curves. See Figure 8 for an extreme example of a vertical isosceles triangle. Note that while our algorithm is flexible enough to handle sharp corners, we only analyze quality bounds near single curves in section 3 .

\subsection{Handling Hanging Nodes}

Squares with a geometric curve or vertex produced polygons and were meshed with midpoint subdivision. We now consider the remaining empty squares. Squares 


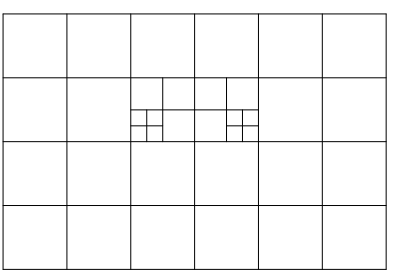

(a) An unblanced quadtree.

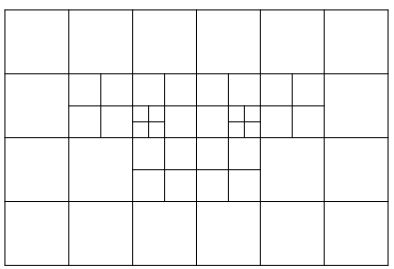

(b) A balanced quadtree.

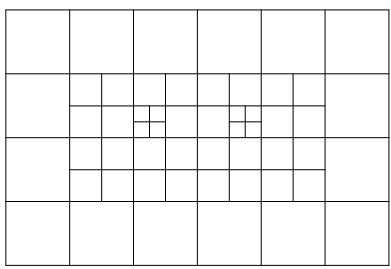

(c) A strongly-balanced quadtree.

Figure 3: (a) An unbalanced quadtree of 3 levels. In (b), a balanced tree is one where no edge is shared between two squares with more than a difference of 1 in levels. In (c), a strongly-balanced tree is one where no point is connected to two squares of more than a difference of 1 in levels. If either condition is violated, the larger square gets refined. We require a strongly-balanced quadtree to enable the 2-ref templates in the last step.

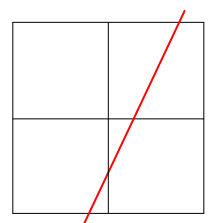

(a) Grid points close to boundary.

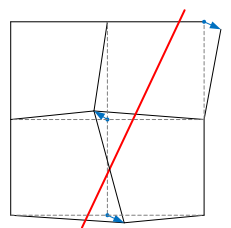

(b) Geometrynormal repelling.

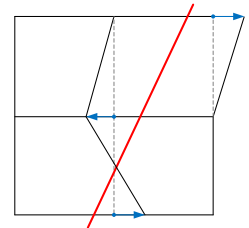

(c) Grid-aligned repelling.

273

274 275

Figure 4: Repelling grid points away from boundary if they are too 280 close to it as in (a). In (b), repelling moves a corner in the direction normal to the boundary until its Euclidean distance is exactly $\delta$. In (c), the curve has slope larger than one and is considered vertical. Therefore, repelling moves corners horizontally along grid lines until 283 they are $\delta$ away in the horizontal direction.

with no hanging nodes are immediately mesh quads. 285 Squares with hanging nodes are meshed using the two- 286 refinement (2-ref) templates with corner marking [35]. ${ }_{287}$ The two-refinement templates shown in Figure 7 ( a) split ${ }_{288}$ an element with a hanging node into either three or four 289 quads. Using a checker board pattern, we mark square 290 corners for template application. In essence, the mark- 291 ing provides a local and globally consistent way to pair ${ }_{292}$ adjacent squares with one or three hanging nodes to- ${ }_{293}$ gether, providing an all-quad mesh that allows size tran- 294 sitions. For example, the strongly-balanced quadtree 295 with hanging nodes in Figure 3 is marked for 2-ref tem- 296 plate application in Figure 7 (b) and consequently re- 297 fined in Figure 7 (c).

\section{Analysis}

In this section, we analyze the theoretical bounds on ${ }^{302}$ the element quality in terms of angle bounds and edge 303 lengths. We denote the maximum angle: $\omega$, the mini- 304 mum angle: $\alpha$, the maximum edge length: $e_{\max }$, and the 305 minimum edge length: $e_{\min }$. We take several cases into 306 consideration, proceeding from simple to hard cases, to 307 analytically study the impact of repelling and mid-point subdivision. We do not analyze the cases of a sharp input angle at a vertex: the achieved angles and edge lengths depend on this angle.

\subsection{Squares without Geometry or Moved Corners}

Quadtree squares that do not interact with the geometry are meshed into quads using templates, depending on the hanging nodes. It is trivial to see that these results in edge lengths between $s$ and $s / 2$ (for a side with a hanging node) and angles between $45^{\circ}$ and $135^{\circ}$, as can be seen in Figure 7 (a).

\subsection{Squares with Moved Corners, without Geometry}

Corners of a square with no hanging nodes would be moved in our algorithm using either boundary-normal repelling, or grid-aligned repelling. Each approach results in slightly different angle bounds. Figures 9 and 10 shows the geometry of the largest and smallest possible angles at a corner after repelling by $\delta$. The edge length $|e|$ varies in both repelling cases between $s-2 \delta$ and $s+2 \delta$. Next, we derive formulas for the expected minimum and maximum angles, and quantify their values for the experimental choice of $\delta=s / 4$ :

Boundary-Normal Repelling. Starting at Figure 9 (a), the largest angle would be formed when a corner, say $q$, moves towards the square's center a distance $\delta$ in the direction $\overrightarrow{q p}$, while its neighbor corners $v$ and $t$ move a distance $\delta$ outwards in directions such that $q v$ and $q t$ are orthogonal to the displacements of $v$ and $t$, respectively. In this case, as shown in Figure 9 (b), the horizontal distance between $q$ and $v$ is $|u q|=s-\frac{\delta}{\sqrt{2}}-\delta \cos \kappa$, while the vertical distance $|u v|=\frac{\delta}{\sqrt{2}}+\delta \sin \kappa$. This yields an angle $\gamma$ between $v q$ and $q u$, where $\tan \gamma=|u v| /|u q|$. The maximum angle $\omega$ at corner $q$ is therefore equal to $90^{\circ}+2 \gamma$. When $\delta=s / 4$, the maximum angle is $\omega=148.8^{\circ}$. 


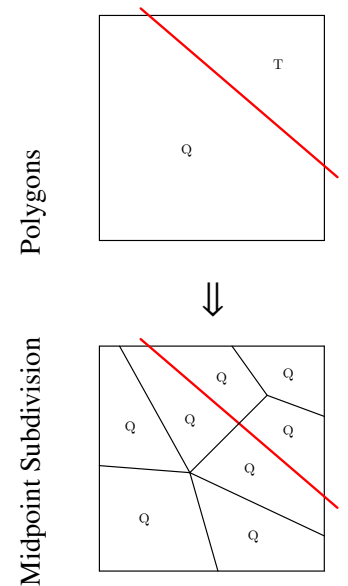

(a)

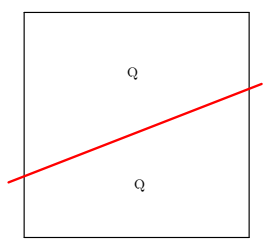

$\Downarrow$

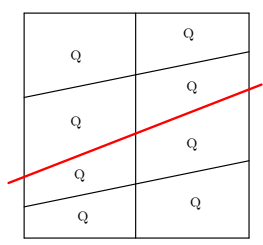

(b)

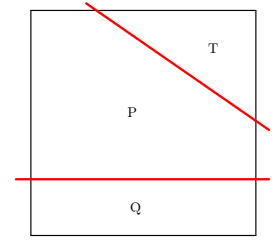

$\Downarrow$

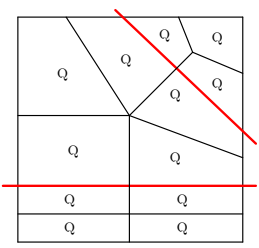

(c)

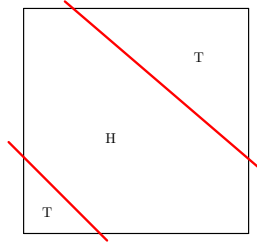

$\Downarrow$

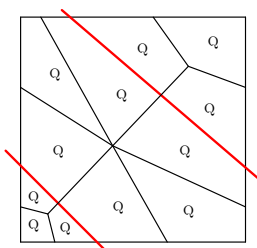

(d)

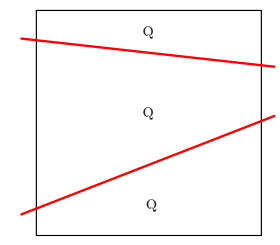

$\Downarrow$

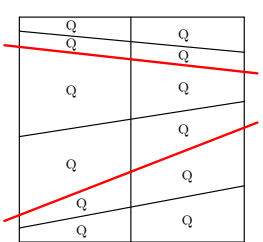

(e)

Figure 5: Example grid-geometry intersection and the resulting midpoint subdivision mesh. Input geometry curves split squares into polygons: Triangles $(\mathrm{T})$, Quads $(\mathrm{Q})$, Pentagons $(\mathrm{P})$, or Hexagons $(\mathrm{H})$. Each polygon is divided into quad elements. The top row shows intersected elements, while the bottom shows the split and divided mesh. Columns show the cases when the boundary splits a quad element into (a) a triangle and a pentagon, (b) two quads, (c) a triangle, a quad, and a pentagon, (d) two triangles and a hexagon, and (e) three quads.

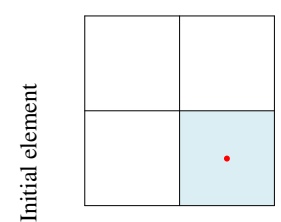

$\Downarrow$

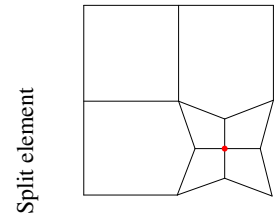

$\Downarrow$

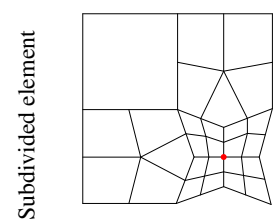

(a)

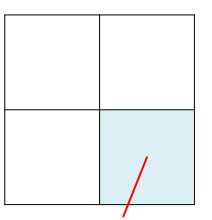

$\Downarrow$

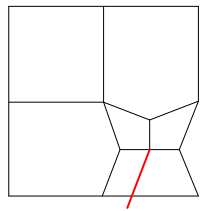

$\Downarrow$

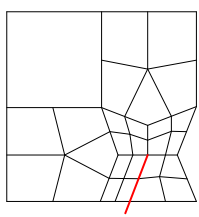

(b)

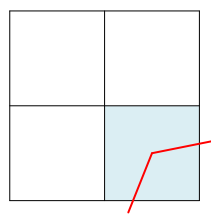

$\Downarrow$

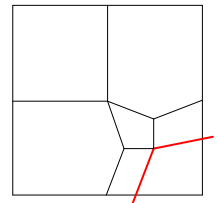

$\Downarrow$

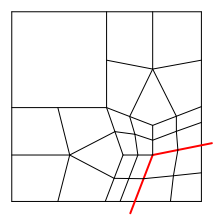

(c)

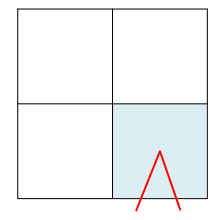

$\Downarrow$

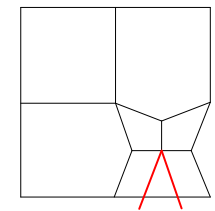

$\Downarrow$

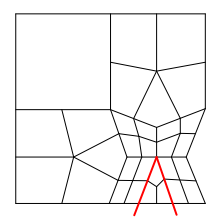

(d)

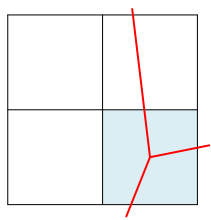

$\Downarrow$

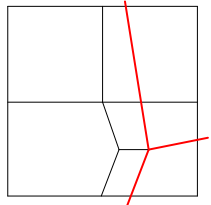

$\Downarrow$

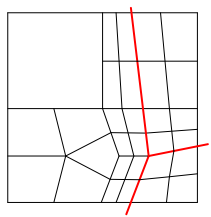

(e)

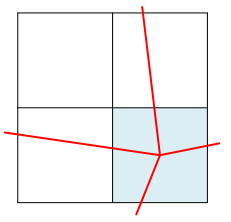

$\Downarrow$

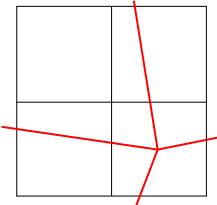

$\Downarrow$

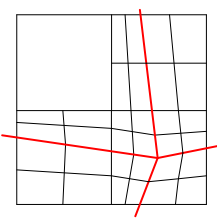

(f)

Figure 6: Refining a square containing a geometric vertex into all-quad elements: (a) an isolated vertex. (b) a hanging edge, (c) and (d) vertex with two line segments, (e) vertex with three curves, (f) vertex with four curves. Other intersection scenarios can be decomposed into superpositions of these cases. The square is first split into four quad elements, with pulling the new corners closer to the vertex to avoid the creation of a flat angle. Then, these quads and the squares sharing an edge with the initial element get split into quad elements by midpoint subdivision. This generates nearby squares with hanging nodes, which are resolved in the last step of the algorithm. 


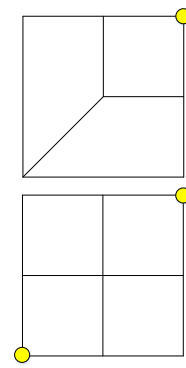

(a) Templates

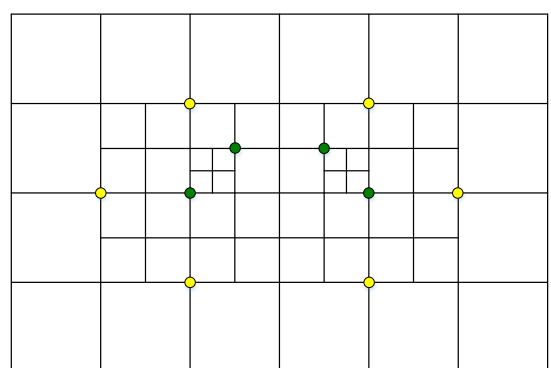

(b) A quadtree with hanging nodes.

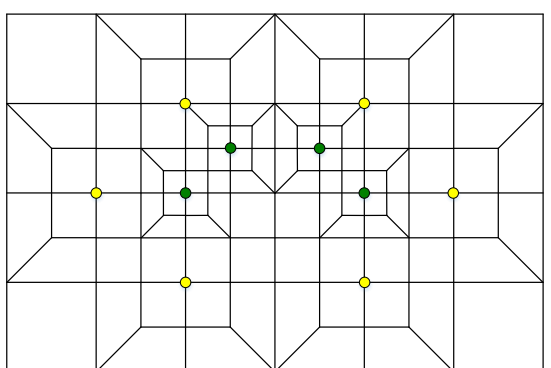

(c) A balanced quadtree with templates applied

Figure 7: Applying the two-refinement templates to the strongly balanced quadtree with hanging nodes in Figure 3 The two-refinement templates are shown in (a), and applied to the marked mesh points in (b) to eliminate the hanging nodes as in (c).

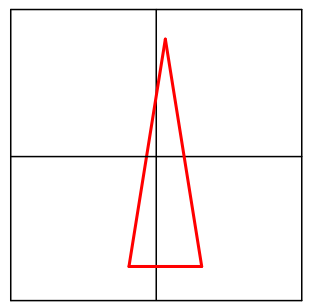

(a) An extreme sharp corner case of an isosceles triangle.

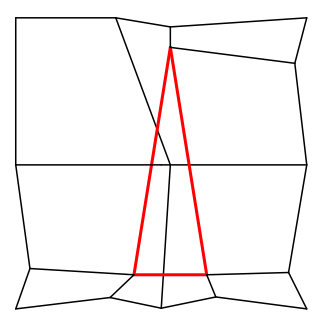

(c) Splitting elements.

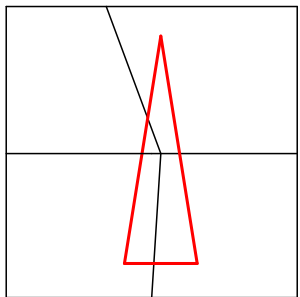

(b) Trapped point is centered half 326 way between goemetry curves.

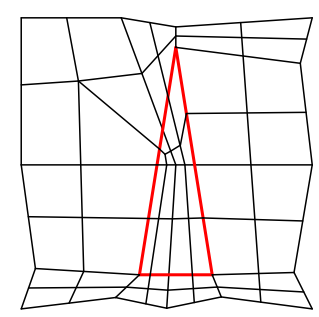

(d) Subdividing elements.

Figure 8: Applying our all-quad algorithm to a vertical isosceles triangle. The trapped mid-point is centered between the two vertical geometry curves. Our algorithm is flexible enough to handle this sharp corner, but does not provide a quality bound in this case.

On the other hand, the smallest angle would be formed when a corner, say $p$, moves outwards away from the square's center a distance $\delta$ in the direction $q \vec{p}$, while its neighbor corners $t$ and $v$ move a distance $\delta$ towards the square's center in directions such that $p t$ and $p v$ are orthogonal to the displacements of $t$ and $v$, respectively. In this case, as shown in Figure 9 (c), the horizontal distance between $p$ and $t$ is $|u p|=s+\frac{\delta}{\sqrt{2}}-\delta \cos \theta$, while the vertical distance $|u t|=\frac{\delta}{\sqrt{2}}+\delta \sin \theta$. This yields

an angle $\beta$ between $p u$ and $p t$, where $\tan \beta=|u t| /|u p|$. The minimum angle $\alpha$ at corner $p$ is therefore equal to $90^{\circ}-2 \beta$. When $\delta=s / 4$, the minimum angle is $\alpha=48.67^{\circ}$.

Grid-Aligned Repelling. This repelling approach can be perceived as a special case of the boundary normal repelling that limits the points' movements to the grid lines. Similar to the analysis above, we start with an initial square as shown in Figure 10(a). The largest angle would be formed when two opposite corners, say $v$ and $t$ move a distance $\delta$ outwards from the square on the vertical and horizontal grid lines, respectively, and the corner $q$ moves a distance $\delta$ on either grid line. In this case, as shown in Figure 10(b), the largest angle can be found to be $\omega=90^{\circ}+\tan ^{-1}\left(\frac{\delta}{s-\delta}\right)+\tan ^{-1}(2 \delta / s)$. When $\delta=s / 4, \omega=135^{\circ}$.

On the other hand, the smallest angle would be formed when two opposite corners, say $v$ and $t$ move a distance $\delta$ inwards, on the horizontal and vertical grid lines, respectively, and corner $p$ moves a distance $\delta$ on either grid line. In this case, as shown Figure 10 (c), the smallest angle can be found to be $\alpha=90^{\circ}-\tan ^{-1}\left(\frac{\delta}{s+\delta}\right)-$ $\tan ^{-1}(2 \delta / s)$. When $\delta=s / 4, \alpha=52.125^{\circ}$.

The bounds on the angles at the center node of the templates are better than the above corner-angle bounds. The template for two hanging nodes may split a corner angle. The angle bounds resulting from either repelling approach are then $\left(48.67^{\circ}, 148.8^{\circ}\right)$ and the edge length bounds are $(0.5,1.5) s$.

\subsection{Squares with Geometry}

Squares with geometry are divided into 3-6 sided polygons by the curves of the geometry. Some corners may be moved away from the geometry to ensure a $\delta$ clearance. 


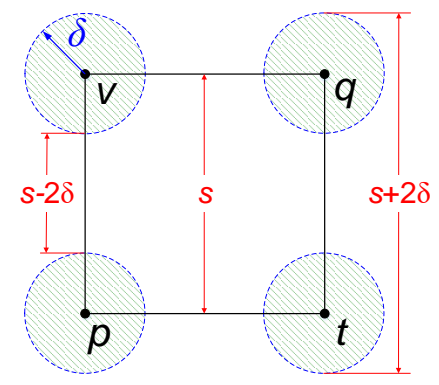

(a) Possible boundary-normal corner movements.

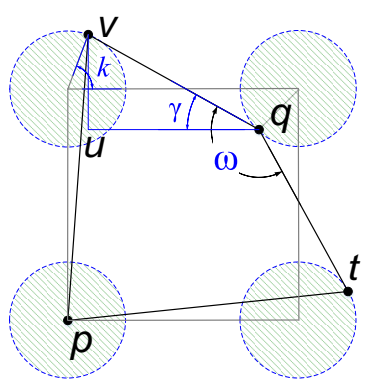

(b) Corner movements for the largest possible angle.

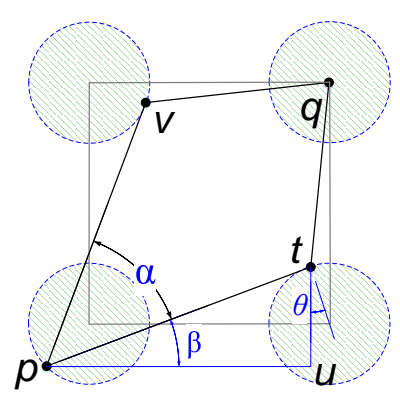

(c) Corner movements for the smallest possible angle.

Figure 9: Angles and edge lengths for boundary-normal repelling corners of a square.

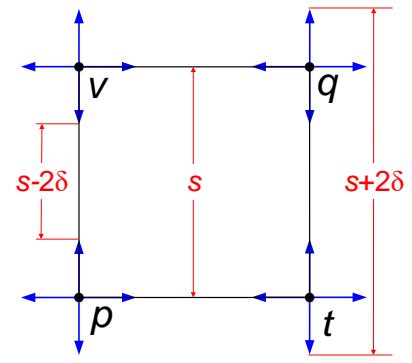

(a) Possible grid-aligned corner movements.

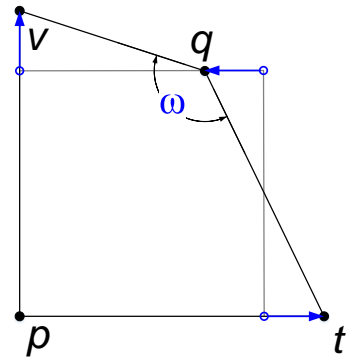

(b) Corner movements for the largest possible angle.

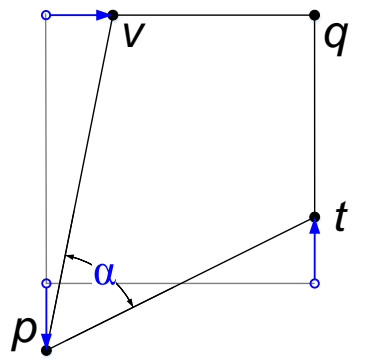

(c) Corner movements for the smallest possible angle.

Figure 10: Angles and edge lengths for grid-aligned repelling corners of a square.
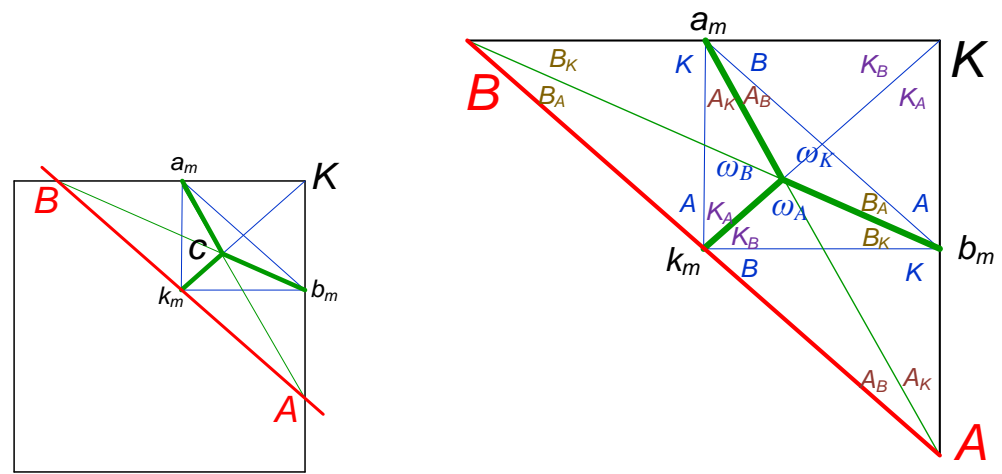

Figure 11: Angles in a triangular polygon split into quads along segments from corners to midpoints.

\subsubsection{Edge Lengths Bounds}

For a square with a vertex, a moved side length is at ${ }_{358}$ most $s+2 \delta$, bounded by the case of a square corner- 359 corner edge with both nodes moved away from each ${ }_{360}$ other by $\delta$. For a square cut only by curves, we conwell). Since curves are straight, two adjacent square corners are moved in the same direction. If a single corner is moved, then we have $|e|^{2}<s^{2}+\delta^{2}$. An edge 
along a curve may not be longer than square diagonal, 406 $|e|<\sqrt{2} s$. Any corner-corner distance is at least $\delta, 407$ and hence corner-midpoint edge is at least $\delta / 2$. Any 408 vertex center to midpoint edge is at least $\delta$, by construc- 409 tion. We now consider centers that are not vertices. Any 410 polygon center is at least $\delta / 3$ from a non-center edge by ${ }_{411}$ the following. The worst case is a triangle. Let one ${ }_{412}$ edge be the horizontal axis. The other corner must be at ${ }_{413}$ least $\delta$ from the edge. Since the center is placed at the ${ }_{414}$ geometric center, in particular its vertical coordinate is 415 one-third of each of the corners' vertical coordinate, its ${ }_{416}$ height above the edge is at least $\delta / 3$. To summarize, for ${ }_{417}$ $\delta=s / 4$,

- corner-midpoint curve edges $|e| \in s[0.125,0.71] \quad 419$

- and side edges $|e| \in s[0.125,0.52]$;

- center-midpoint edges $|e| \geq s / 12$

\subsubsection{Square Corner Angles}

The square corner angles are bounded similar to the uncut squares case, where $\alpha=48.6^{\circ}$, and $\omega=148.8^{\circ}$.

- square corner angles angles $\in\left(48.6^{\circ}, 148.8^{\circ}\right)$.

\subsubsection{Cut Corner Angles}

The limiting case for angles at a node where a curve crosses a square side occurs when each corner moves $\delta$ away from the curve, and the pre-cut side is at most $e_{\max }$ for sides, $1.03 s$. We see that $\sin \alpha=2 \delta / e_{\max }$. Hence for $\delta=\frac{s}{4}$,

- cut corner angles are $\in\left(29^{\circ}, 151^{\circ}\right)$.

\subsubsection{Center and Midpoint Angles}

We consider each case of the number of sides of a polygon.

Triangle. We first consider a triangle, that is a curve 440 cutting off a corner of a square. See Figure 11. The ${ }_{441}$ worst case is when $K$ is already $\delta$ away from the curve ${ }_{442}$ before movement, and the curve is as close to $K$ as pos- ${ }_{443}$ sible on one square side, and as far away as possible on 444 the other. We place center $c$ at $1 / 3$ of the way from $k_{m}{ }_{445}$ to $K$, which is $2 / 3$ of the way to $D$. (This is the trian- ${ }_{446}$ gle centroid, the average of its corners, and where all ${ }_{447}$ three of the corner-to-midpoint lines meet.) From sim- 448 ilar triangles we see $\angle a_{m} b_{m} k=\angle A B c=B_{A}$, etc., as 449 labeled in the figure. Hence $\omega_{K}=180^{\circ}-A_{B}-B_{A}$, 450 $\omega_{A}=180^{\circ}-C_{B}-B_{C}$ and $\omega_{B}=180^{\circ}-A_{K}-K_{A}$. Us- ${ }_{451}$ ing the cotangent angle relationships for midpoint sub- ${ }_{452}$ divided triangles (e.g. section A.3.2 in [36, 37]), we ${ }_{453}$ have $\cot K_{B}=\cot B+2 \cot K$, etc. Given $\{A, B, C\}$, 454 these allow us to derive $A_{B}$, etc., and hence the $\omega$ 's. We consider two extreme cases. The first is isosceles with $|\overline{K B}|=|\overline{K A}|$. Then $\omega_{B}=\omega_{A} \approx 108^{\circ}$, and $\omega_{K} \approx 143.2^{\circ}$. The second case is extreme disparity in edge lengths, $|\overline{K B}|=s$ and $|\overline{K A}|=\delta$. (If $K$ was repelled, then $\overline{K A}$ may be longer, but the resultant angles are less extreme.) Then $\omega_{A} \approx 158.8^{\circ}$, and $\omega_{B} \approx 40.6^{\circ}$ and $\omega_{K} \approx 160.6^{\circ}$.

We bound midpoint angles in the same way. We see that the midpoint angles are $\left\{A+B_{A}, K+B_{K}, B+A_{B}, K+\right.$ $\left.A_{K}, B+K_{b}, A+K_{A}\right\}$. Again considering the extreme edge lengths, we have $\left\{\angle a_{m}, \angle b_{m}\right\} \in\left(26.5^{\circ}, 153.5^{\circ}\right)$. Also $\angle k_{m} \in\left(28^{\circ}, 152^{\circ}\right)$.

For triangles,

- center angles are in $\left(40.6^{\circ}, 160.6^{\circ}\right)$.

- midpoint angles are in $\left(26.5^{\circ}, 153.5^{\circ}\right)$.

Quadrilateral. This is the case of a square cut by a curve from one side to its opposite. Observe that the center is placed where the two lines connecting opposite midpoints meet. (This is not the center of mass in general, but is the average of the four corners.) The worst angles occur at the midpoint of the curve and the cut corners; see Figure 13 The smallest angle is $\gamma$, and the largest angle is its supplement $\bar{\gamma}$. The worst case is when neither quadrilateral corner was moved, but the curve is $\delta$ away from one of the corners, and almost passes through the corner of square on the other side of the curve. In this case the curve has maximum slope $3 / 4$, so $\gamma \geq \arctan (4 / 3)$, and

- center and midpoint angles $\in\left(53.1^{\circ}, 126.9^{\circ}\right)$.

Pentagon. Here we consider a square cut by a single curve. One of the square diagonals does not intersect the curve. We place the center $c$ at the centroid of the triangle $\triangle A K B$ on the far side of that diagonal from the curve. See Figure 12

Consider the corner and midpoint angles. The worst case is when $A$ and $B$ have been repelled $\delta$ from the curve, so we must first bound angles $A, B$, and $K$. For geometry-normal repelling, Figure 14(a), we have $K \leq 90+2 \theta$ where $\theta \leq 12.2^{\circ}$, or $K<114.3^{\circ}$ and $\{\angle B A K, \angle A B K\}>32.8^{\circ}$. (Note $\angle B A K$ and $\angle A B K$ are not mesh angles.) Using the cotangent relations, $\omega_{K} \leq$ 155.7, and the midpoint angles along $\overline{K A}$ and $\overline{K B}$ are in $\left(44.9^{\circ}, 135.1^{\circ}\right)$. (For axis-aligned repelling, we have $K \leq 90^{\circ}+\theta_{2} \leq 104.1^{\circ}$, and the bounds are subsumed. See Figure 14(b).)

Consider the center angles. For $\omega_{A}$, the worst case is when there is no repelling. See Figure 12a, where $\omega_{A}<\angle a_{m} c k_{m}<108.5^{\circ}$. The same holds for $\omega_{B}$. For the other two central angles, we note that the midpoint 


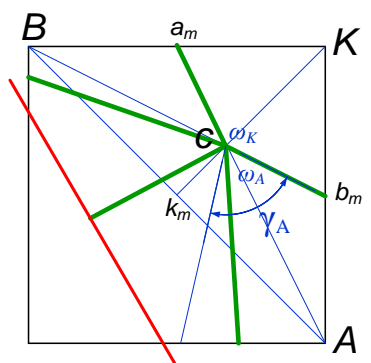

(a) Midpoint subdivision and $\omega_{A}$.

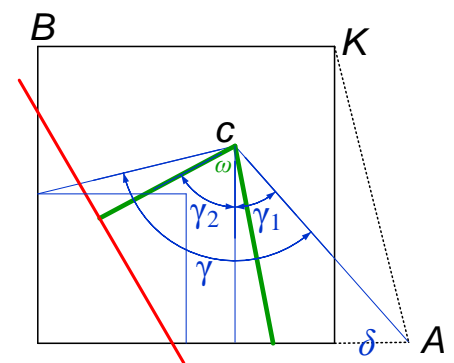

(b) Max $\omega$ opposite a cut corner.

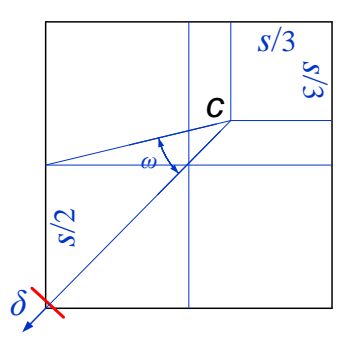

(c) Min $\omega$ opposite a cut corner.

Figure 12: Pentagon midpoint subdivision, and worst-case center angles $\omega$.

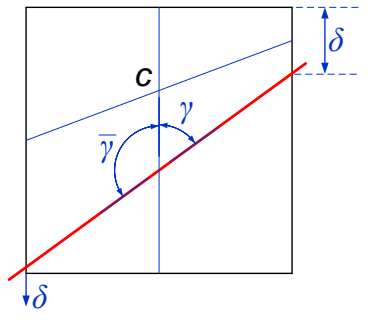

Figure 13: The worst-case angles in a quadrilateral polygon.

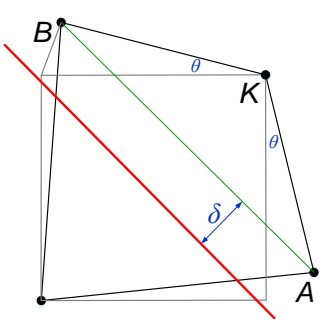

(a) Boundary-normal repelling.

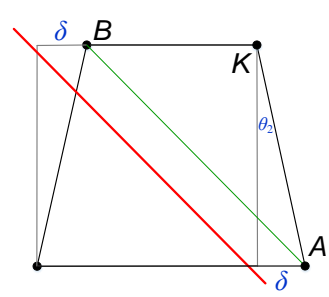

(b) Grid-aligned repelling.
Figure 14: Worst case corner angles for a pentagon. of the curve edge must be inside the lower-left quadrant 486 in Figure 12b Hence $\omega<\gamma=\gamma_{1}+\gamma_{2}<76^{\circ}+41.2^{\circ}=487$ $117.2^{\circ}$.

For the minimum center angles, we have $\omega_{A} \geq A_{K}>489$ $48.3^{\circ}$. The same holds for $\omega_{B}$. For the central angles 490 involving the curve, the worst case is when the curve 491 barely cuts the box corner, see Figure $12 \mathrm{c}$. The angle 492 subtends nearly half of a box length, and $\omega>30.9^{\circ}$. For ${ }^{493}$ the curve and the sides cut by the curve, the midpoint ${ }^{494}$ angles are in $45^{\circ}, 135^{\circ}$.

For pentagons,

- center angles are in $\left(30.9^{\circ}, 155.7^{\circ}\right)$.
- midpoint angles are in $\left(44.9^{\circ}, 135.1^{\circ}\right)$.

Two or more curves in a square. The regions bounded by one curve and the square have meshes and angle bounds as described in the triangle and square cases. It remains to consider the region between two curves: a square, pentagon, or hexagon. See Figure 5 . These cases only occur near sharp angles, and the obtained bounds depend on the input angles. We do not analyze these explicitly, but observe reasonable quality compared to the element containing the sharp vertex.

\subsection{Quality Bound Summary}

Angles are provably bounded away from $0^{\circ}$ and $180^{\circ}$, and edge lengths are within a reasonable constant factor of square side lengths, except perhaps in the presence of input curves that meet at a sharp angle.

- Mesh angles are in $\left(24.3^{\circ}, 160.6^{\circ}\right)$.

- Mesh edge lengths are in $s(0.08,1.5)$.

\section{Experimental Results}

To illustrate the capabilities of our algorithm, we apply it to some domains with smooth and sharp features. Figures 15, 16, 17, 18, and 19 show initial uniform meshes and final all-quad meshes applied to a few smooth domains (flower-, face-, cat-, lake-, and two circles-shaped domains). Our algorithm generates angles that are mostly $90^{\circ}$ and are well-bounded between $45^{\circ}$ and $135^{\circ}$. On the other hand, although our analysis focused on smooth geometries, we show some experimental results applying our algorithm to geometries with sharp corners. In specific, Figures 20, 21, 22, and 23 show initial uniform meshes and final all-quad meshes applied to a few domains with connected regions and sharp features (batman-, star-, double hex-, 
and five holes-shaped domains). Most but not all an- 549 gle are bounded between $45^{\circ}$ and $135^{\circ}$ in these cases as ${ }_{550}^{50}$ shown in the histograms.

Quality metrics of the resulting meshes are summarized in Table 1, including minimum and maximum an- 554 gles, and the minimum and average ratios of shortest to ${ }^{555}$ longest edge lengths in a quad.

\section{Conclusions}

We described an algorithm for all quad meshing of ${ }^{562}$ non-convex domains, with connected regions, conform- ${ }^{56}$ ing to both the interior and exterior of the domain. Our ${ }_{565}$ algorithm is robust and provably-correct. It does not 566 require post-processing cleanup operations, such as pil- 567 lowing, to control the angle bounds of the final mesh, ${ }^{568}$ and can easily handle smooth shapes as well as domains ${ }^{569}$ with sharp features and corners. Our next steps are to ${ }^{570}$ adaptively vary the sizing function along the boundary. ${ }_{572}$ We also seek to demonstrate the method on domains 573 with many curves meeting at a single vertex at sharp 574 angles. A natural extension of the presented algorithm 575 is to apply the same steps to $3 d$ all-hex meshing. This ${ }^{576}$ is simply doable because the steps of our algorithm (re- ${ }^{577}$ pelling, splitting, mid-point subdivision, 2-ref template ${ }_{579}^{578}$ employment) can be employed in $3 \mathrm{~d}$ in a similar fash- 580 ion, especially for smooth surfaces. Some cases of sharp 581 features would certainly be more challenging. The ex- 582 tent of the $3 \mathrm{~d}$ analysis and implementation is outside the ${ }^{583}$ scope of this paper and will therefore be addressed in an ${ }^{584}$ upcoming publication.

\section{Acknowledgements}

Sandia National Laboratories is a multi-program labora- ${ }^{590}$ tory managed and operated by Sandia Corporation, a wholly 592 owned subsidiary of Lockheed Martin Corporation, for the 593 U.S. Department of Energy's National Nuclear Security Ad- ${ }^{594}$ ministration under contract DE-AC04-94AL85000.

\section{References}

[1] K. Ho-Le, Finite element mesh generation methods: a review 600 and classification, Computer-aided design 20 (1) (1988) 27-38.

[2] V. D. Liseikin, Grid generation methods, Springer Science \& Business Media, 2009.

[3] W. Hackbusch, Multi-grid methods and applications, Vol. 4, ${ }^{604}$ Springer-Verlag Berlin, 1985.

[4] K. Lee, Principles of CAD/CAM/CAE systems, Addison- ${ }^{606}$ Wesley Longman Publishing Co., Inc., 1999.

[5] A. Thakur, A. G. Banerjee, S. K. Gupta, A survey of CAD model 608 simplification techniques for physics-based simulation applica- 600 tions, Computer-Aided Design 41 (2) (2009) 65-80.

[6] M. L. Brewer, L. F. Diachin, P. M. Knupp, T. Leurent, D. J. ${ }^{61}$ Melander, The mesquite mesh quality improvement toolkit., in: 612 IMR, 2003.
[7] R. V. Garimella, M. J. Shashkov, P. M. Knupp, Triangular and quadrilateral surface mesh quality optimization using local parametrization, Computer Methods in Applied Mechanics and Engineering 193 (9) (2004) 913-928.

[8] B. D. Anderson, S. E. Benzley, S. J. Owen, Automatic all quadrilateral mesh adaption through refinement and coarsening, in: Proceedings of the 18th International Meshing Roundtable, Springer, 2009, pp. 557-574.

[9] J. Daniels, C. T. Silva, J. Shepherd, E. Cohen, Quadrilateral mesh simplification, in: ACM Transactions on Graphics (TOG), Vol. 27, ACM, 2008, p. 148.

[10] J. Peters, U. Reif, The simplest subdivision scheme for smoothing polyhedra, ACM Transactions on Graphics (TOG) 16 (4) (1997) 420-431.

[11] H. Prautzsch, Q. Chen, Analyzing midpoint subdivision, Computer Aided Geometric Design 28 (7) (2011) 407-419.

[12] D. Eppstein, Diamond-kite adaptive quadrilateral meshing, Engineering with Computers 30 (2) (2014) 223-235.

[13] S. J. Owen, M. L. Staten, S. A. Canann, S. Saigal, Q-morph: an indirect approach to advancing front quad meshing, International Journal for Numerical Methods in Engineering 44 (9) (1999) 1317-1340.

[14] M. S. Ebeida, K. Karamete, E. Mestreau, S. Dey, Q-tran: a new approach to transform triangular meshes into quadrilateral meshes locally, in: Proceedings of the 19th International Meshing Roundtable, Springer, 2010, pp. 23-34.

[15] T. D. Blacker, M. B. Stephenson, Paving: A new approach to automated quadrilateral mesh generation, International Journal for Numerical Methods in Engineering 32 (4) (1991) 811-847.

[16] J. Zhu, O. Zienkiewicz, E. Hinton, J. Wu, A new approach to the development of automatic quadrilateral mesh generation, International Journal for Numerical Methods in Engineering 32 (4) (1991) 849-866.

[17] D. R. White, P. Kinney, Redesign of the paving algorithm: Robustness enhancements through element by element meshing, in: 6th International Meshing Roundtable, Citeseer, 1997, pp. 323-335.

[18] P. L. Baehmann, S. L. Wittchen, M. S. Shephard, K. R. Grice, M. A. Yerry, Robust, geometrically based, automatic twodimensional mesh generation, International Journal for Numerical Methods in Engineering 24 (6) (1987) 1043-1078.

[19] R. Schneiders, R. Schindler, F. Weiler, Octree-based generation of hexahedral element meshes, in: Proceedings of the 5th International Meshing Roundtable, Citeseer, 1996.

[20] X. Liang, M. S. Ebeida, Y. Zhang, Guaranteed-quality allquadrilateral mesh generation with feature preservation, Computer Methods in Applied Mechanics and Engineering 199 (29) (2010) 2072-2083.

[21] X. Liang, Y. Zhang, Hexagon-based all-quadrilateral mesh generation with guaranteed angle bounds, Computer Methods in Applied Mechanics and Engineering 200 (23) (2011) 20052020.

[22] X. Liang, Y. Zhang, Matching interior and exterior allquadrilateral meshes with guaranteed angle bounds, Engineering with Computers 28 (4) (2012) 375-389.

[23] K. Shimada, J.-H. Liao, T. Itoh, et al., Quadrilateral meshing with directionality control through the packing of square cells., in: IMR, 1998, pp. 61-75.

[24] M. Bern, D. Eppstein, Quadrilateral meshing by circle packing, International Journal of Computational Geometry \& Applications 10 (04) (2000) 347-360.

[25] F. B. Atalay, S. Ramaswami, D. Xu, Quadrilateral meshes with bounded minimum angle, in: Proceedings of the 17th International Meshing Roundtable, Springer, 2008, pp. 73-91.

[26] M. Fisher, P. Schröder, M. Desbrun, H. Hoppe, Design of tan- 


\begin{tabular}{|c|c|c|c|c|c|c|}
\hline Input Domain & $v$ & $E$ & $\theta_{\min }$ & $\theta_{\max }$ & $\alpha_{\min }$ & $\alpha_{\text {avg }}$ \\
\hline \multicolumn{7}{|c|}{ Smooth Geometries } \\
\hline flower & 4150 & 4130 & $45^{\circ}$ & $135^{\circ}$ & 0.107 & 0.705 \\
\hline face & 3580 & 3496 & $45^{\circ}$ & $135^{\circ}$ & 0.332 & 0.714 \\
\hline cat & 5466 & 5417 & $45^{\circ}$ & $135^{\circ}$ & 0.132 & 0.709 \\
\hline lake & 153997 & 153964 & $45^{\circ}$ & $135^{\circ}$ & 0.064 & 0.718 \\
\hline 2 circles & 3220 & 3190 & $45^{\circ}$ & $135^{\circ}$ & 0.108 & 0.690 \\
\hline \multicolumn{7}{|c|}{ Sharp Features Geometries } \\
\hline batman & 26908 & 26747 & $31^{\circ}$ & $145^{\circ}$ & 0.095 & 0.866 \\
\hline star & 11511 & 11410 & $27.2^{\circ}$ & $160.6^{\circ}$ & 0.083 & 0.864 \\
\hline double hex & 23389 & 23298 & $22.1^{\circ}$ & $157.2^{\circ}$ & 0.127 & 0.847 \\
\hline 5 holes & 31773 & 31679 & $22.5^{\circ}$ & $169.7^{\circ}$ & 0.012 & 0.847 \\
\hline
\end{tabular}

Table 1: Mesh quality examples: $v$ is the number of mesh vertices, $E$ is the number of mesh elements, $\theta_{\min }$ and $\theta_{\max }$ are the minimum and maximum angles, $\alpha_{\min }$ is the minimum ratio of shortest to longest edge length in a quad, and $\alpha_{\text {avg }}$ is the average ratio of shortest to longest edges in a quad.

gent vector fields, in: ACM Transactions on Graphics (TOG), Vol. 26, ACM, 2007, p. 56.

[27] D. Cohen-Steiner, J.-M. Morvan, Restricted delaunay triangulations and normal cycle, in: Proceedings of the nineteenth annual symposium on Computational geometry, ACM, 2003, pp. 312321.

[28] F. Cazals, M. Pouget, Estimating differential quantities using polynomial fitting of osculating jets, Computer Aided Geometric Design 22 (2) (2005) 121-146.

[29] F. Kälberer, M. Nieser, K. Polthier, Quadcover-surface parameterization using branched coverings, in: Computer Graphics Forum, Vol. 26, Wiley Online Library, 2007, pp. 375-384.

[30] A. A. Rushdi, S. A. Mitchell, C. L. Bajaj, M. S. Ebeida, Robust all-quad meshing of domains with connected regions, Procedia Engineering (Proceedings of the 24th International Meshing Roundtable) 124 (2015) 96-108.

[31] T. Ju, F. Losasso, S. Schaefer, J. Warren, Dual contouring of hermite data, ACM Transactions on Graphics (TOG) 21 (3) (2002) 339-346.

[32] Y. Zhang, J. Qian, Dual contouring for domains with topology ambiguity, Computer Methods in Applied Mechanics and Engineering 217 (2012) 34-45.

[33] Y. Zhang, C. Bajaj, Finite element meshing for cardiac analysis, Univ. of Texas at Austin: ICES Technical Report.

[34] S. A. Mitchell, T. J. Tautges, Pillowing doublets: Refining a mesh to ensure that faces share at most one edge, 4th International Meshing Roundtable (1995) 231-240.

[35] M. S. Ebeida, A. Patney, J. D. Owens, E. Mestreau, Isotropic conforming refinement of quadrilateral and hexahedral meshes using two-refinement templates, International Journal for $\mathrm{Nu}$ merical Methods in Engineering 88 (10) (2011) 974-985.

[36] S. A. Mitchell, M. A. Mohammed, A. H. Mahmoud, M. S. Ebeida, Delaunay quadrangulation by two-coloring vertices, Procedia Engineering 8 (2014) 364-376, proceedings of the 23rd International Meshing Roundtable. Freely available online at Science Direct

[37] S. A. Mitchell, M. A. Mohammed, A. H. Mahmoud, M. S. Ebeida, Delaunay quadrangulation by two-coloring vertices extended version with quad-quality proofs appendix, Tech. Rep. SAND2014-16625C, Sandia National Laboratories (2014). 


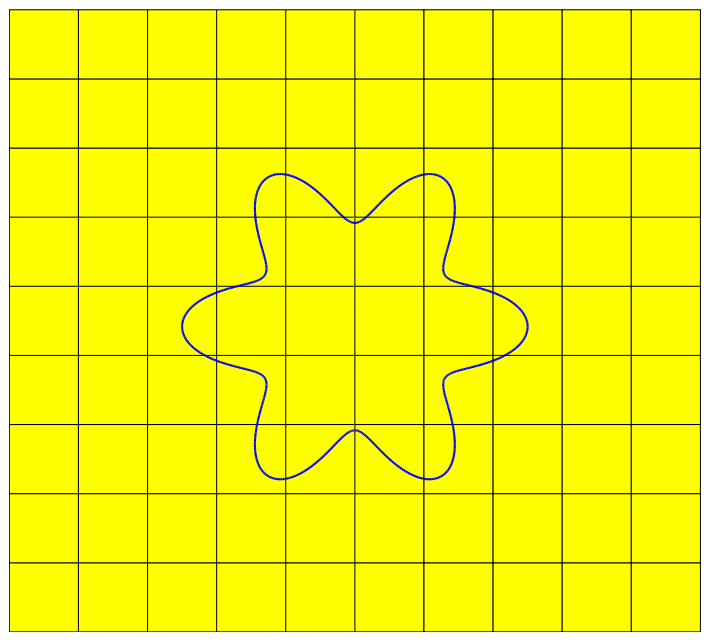

(a) Initial grid and domain.
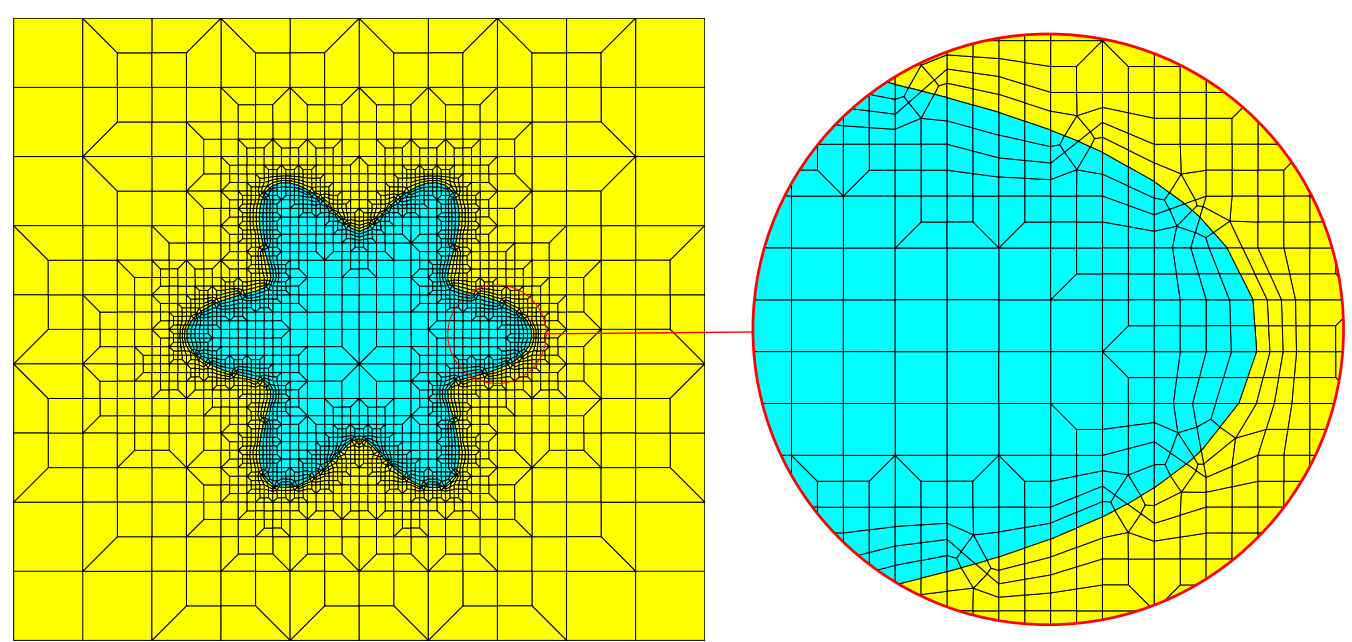

(b) Final all-quad mesh and a magnified region.

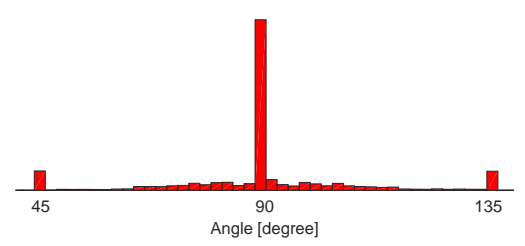

(c) Angle histogram.

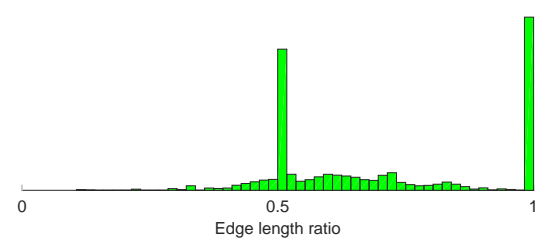

(d) Edge length histogram.

Figure 15: Application of the all-quad meshing algorithm to a valentine flower shaped domain. 


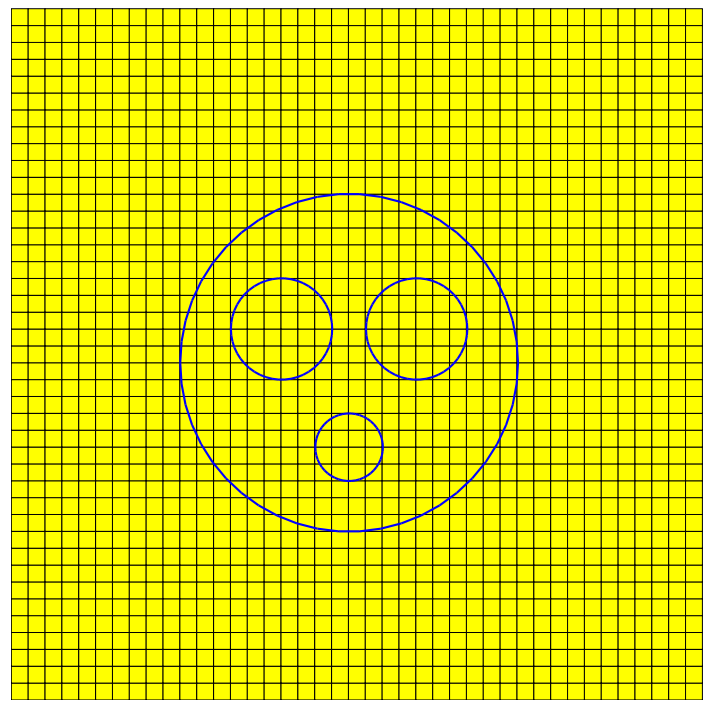

(a) Initial grid and domain.
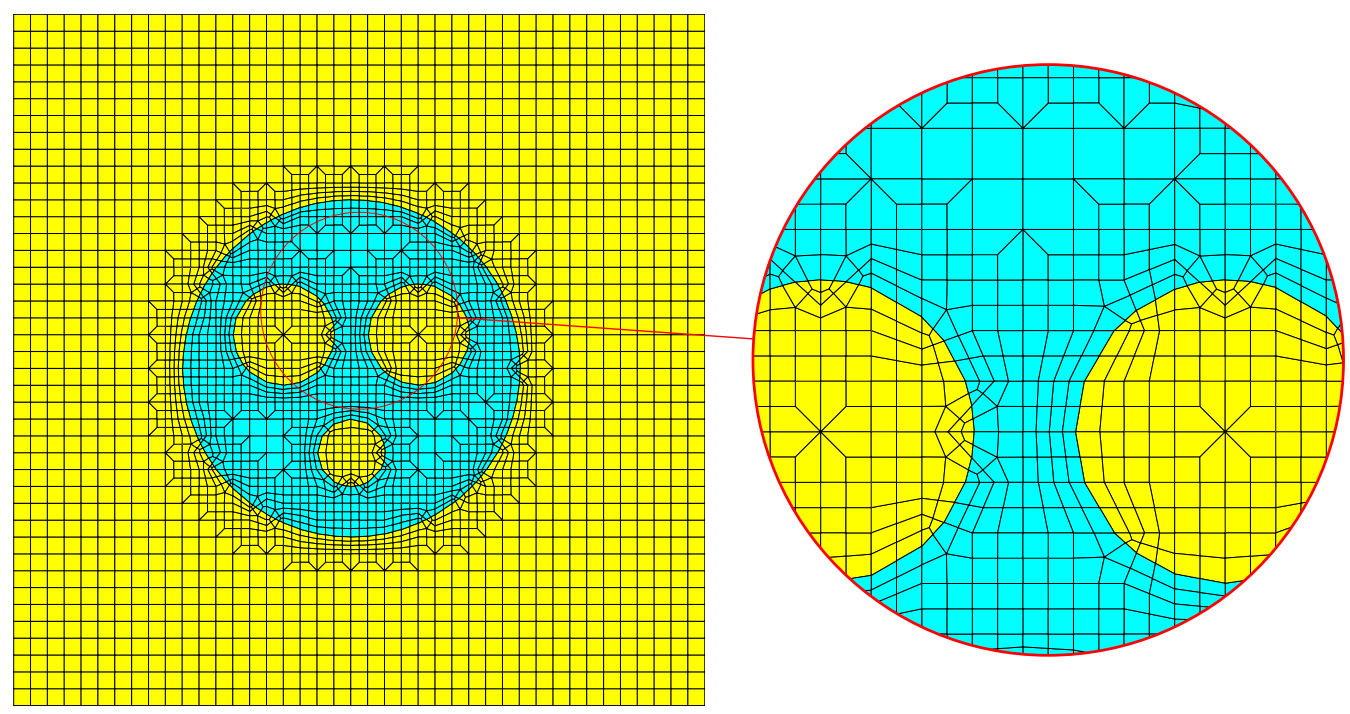

(b) Final all-quad mesh and a magnified region.

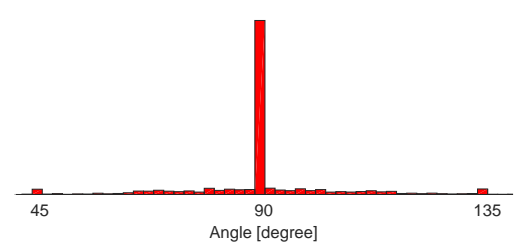

(c) Angle histogram.

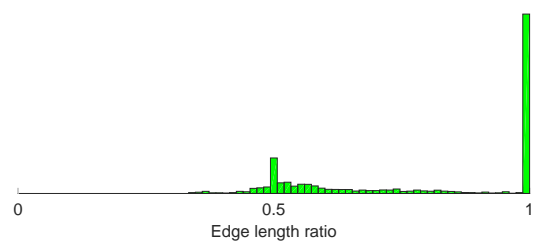

(d) Edge length histogram.

Figure 16: Application of the all-quad meshing algorithm to a face-shaped domain. 


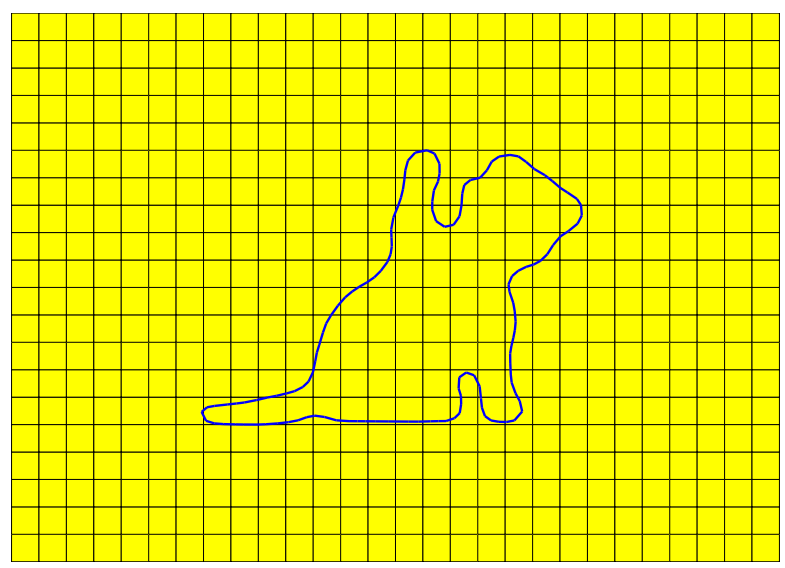

(a) Initial grid and domain.
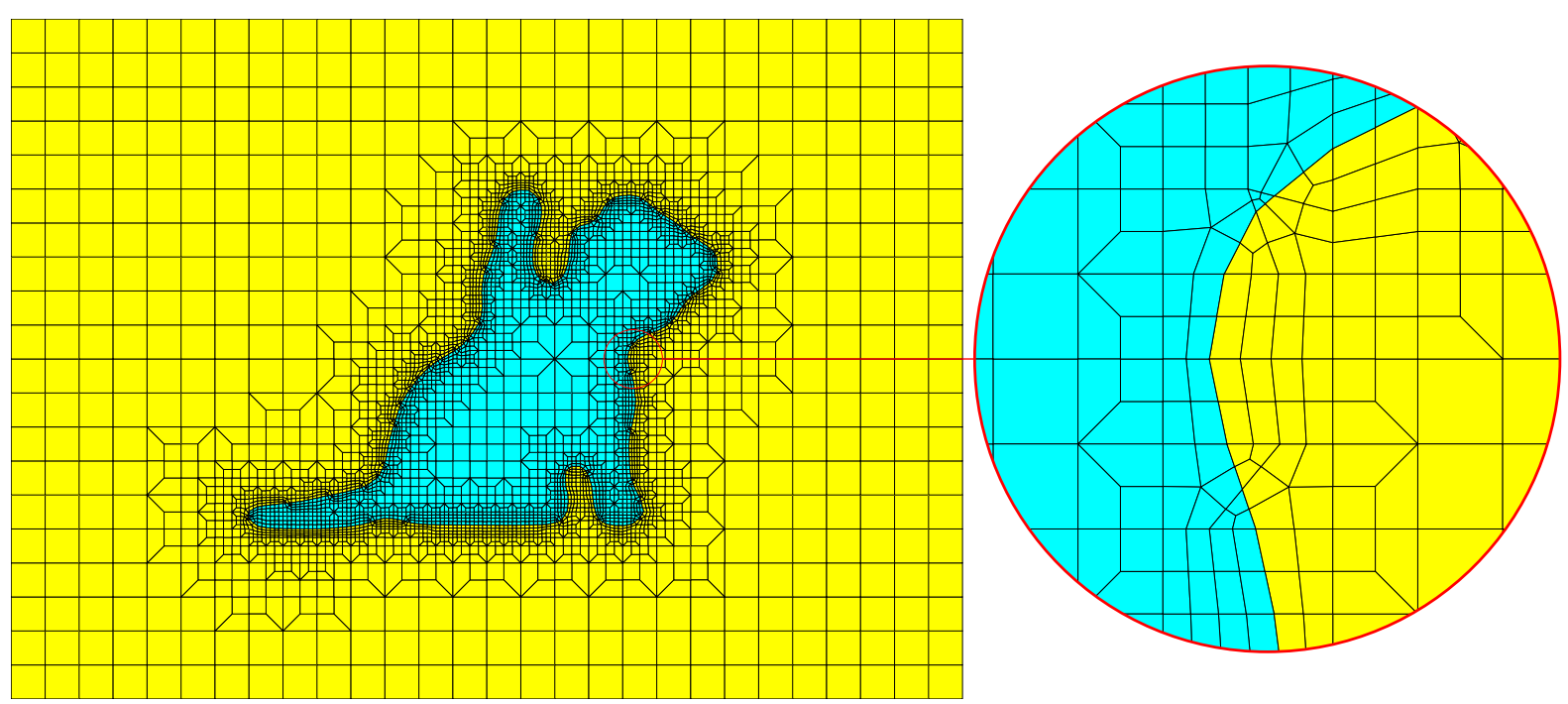

(b) Final all-quad mesh and a magnified region.

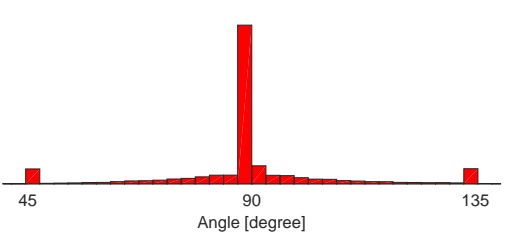

(c) Angle histogram.

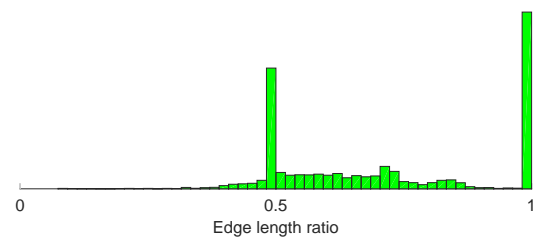

(d) Edge length histogram.

Figure 17: Application of the all-quad meshing algorithm to a cat-shaped domain. 


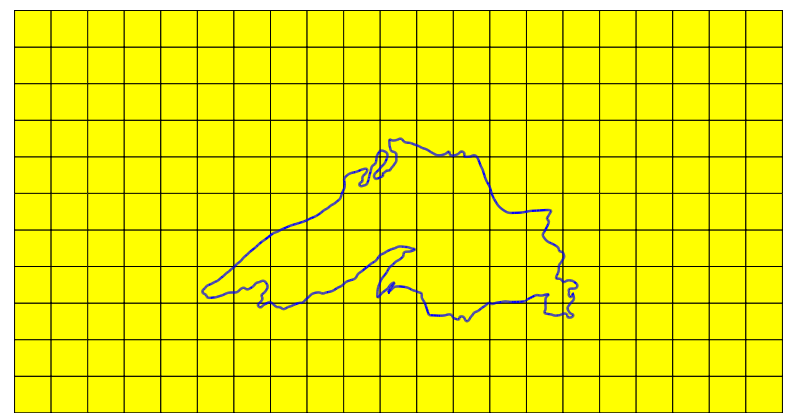

(a) Initial grid and domain.

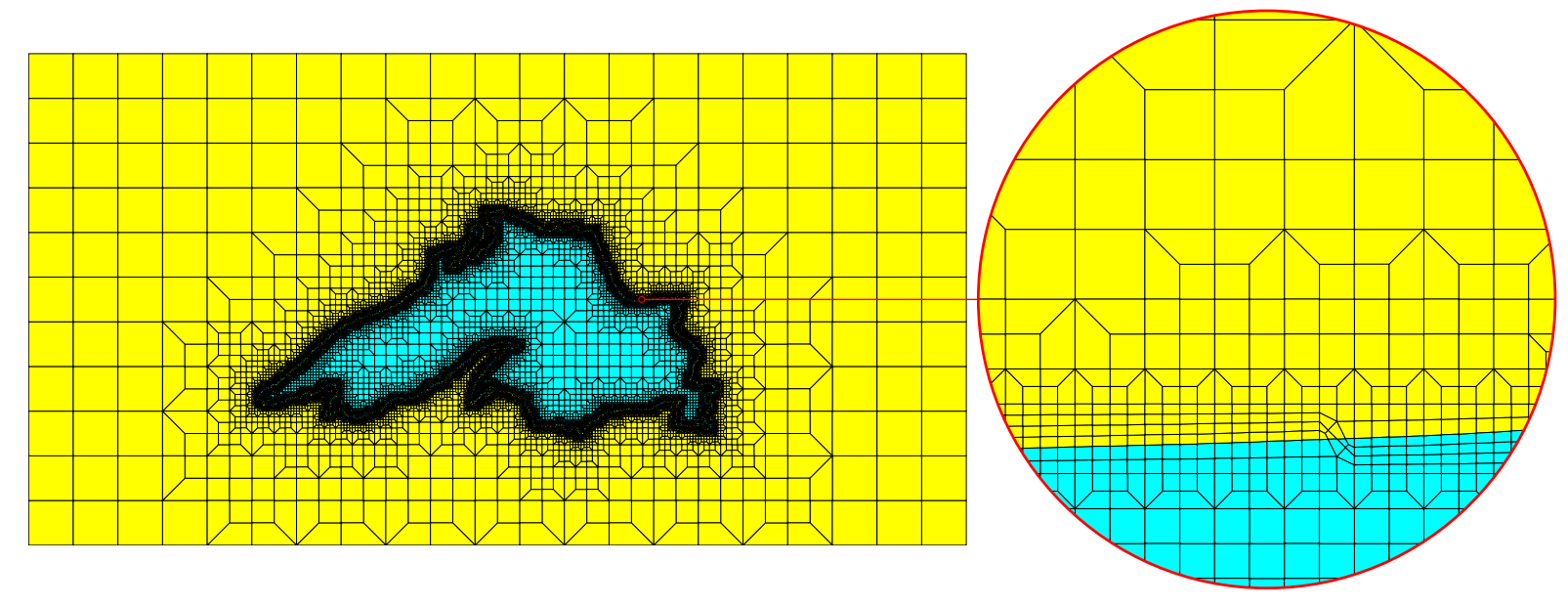

(b) Final all-quad mesh and a magnified region.

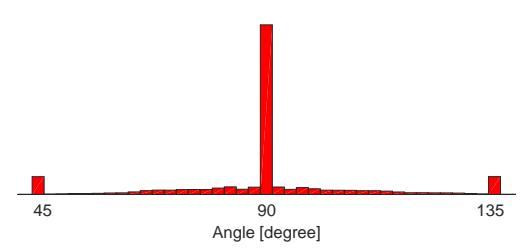

(c) Angle histogram.

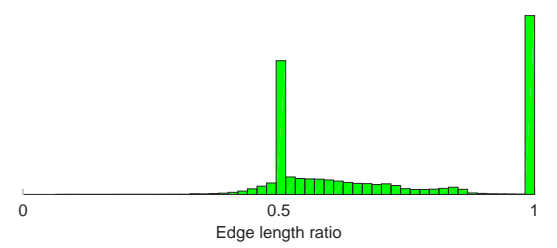

(d) Edge length histogram.

Figure 18: Application of the all-quad meshing algorithm to a lake shaped domain. 


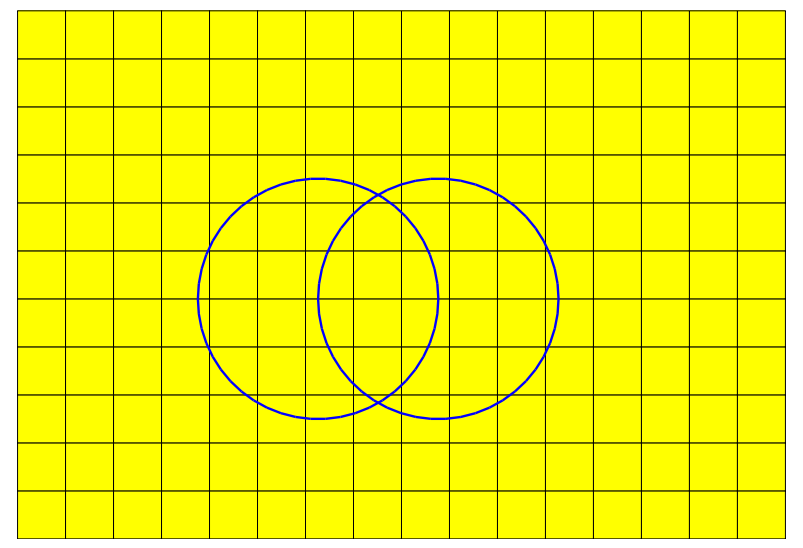

(a) Initial grid and domain.
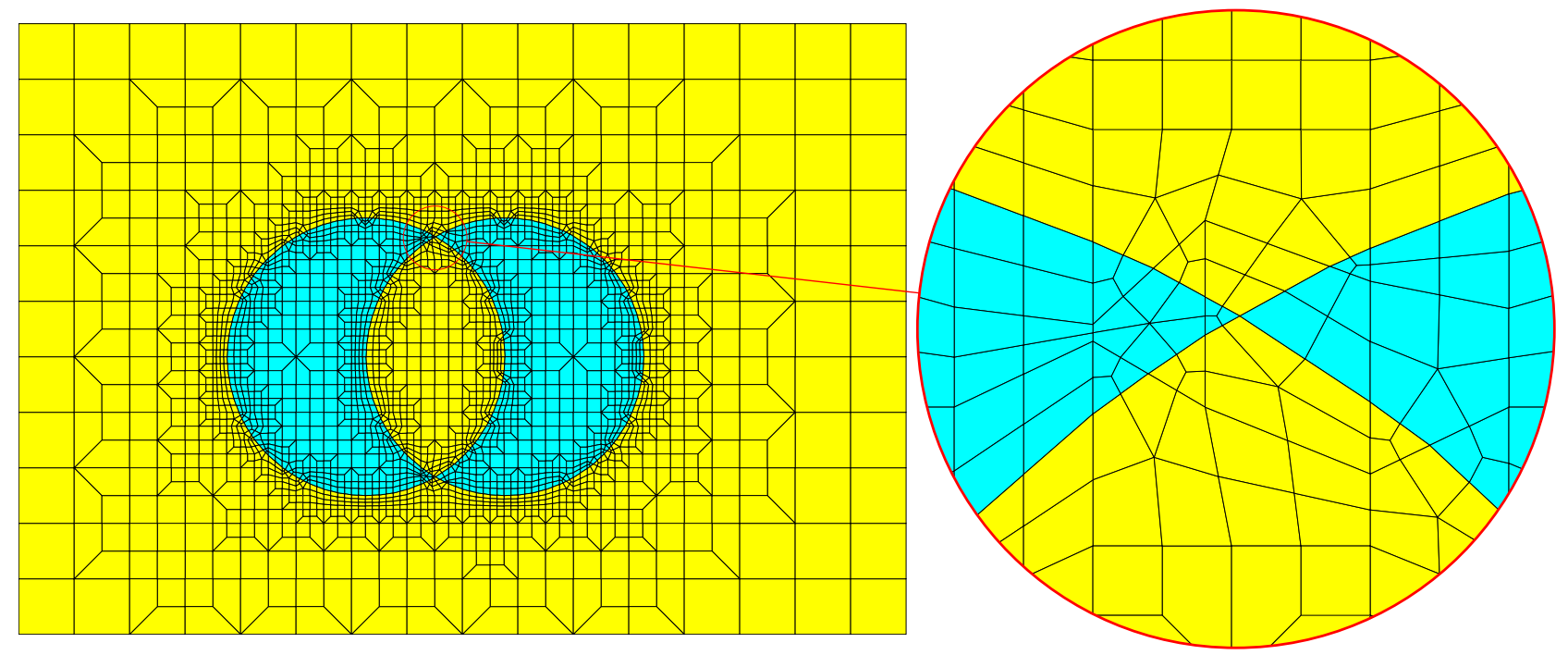

(b) Final all-quad mesh and a magnified region.

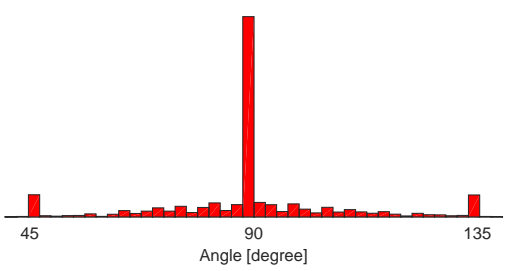

(c) Angle histogram.

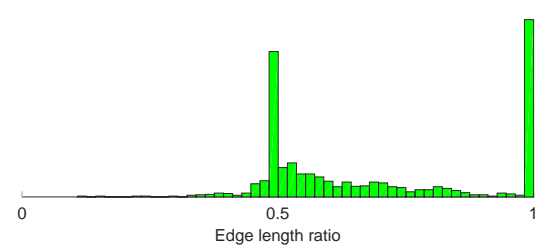

(d) Edge length histogram.

Figure 19: Application of the all-quad meshing algorithm to an intersecting-circles shaped domain. 


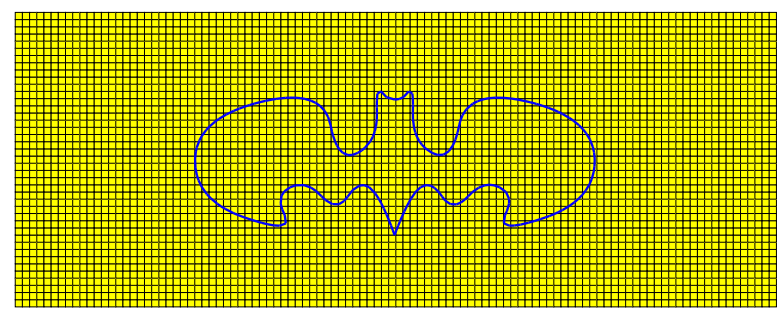

(a) Initial grid and domain.

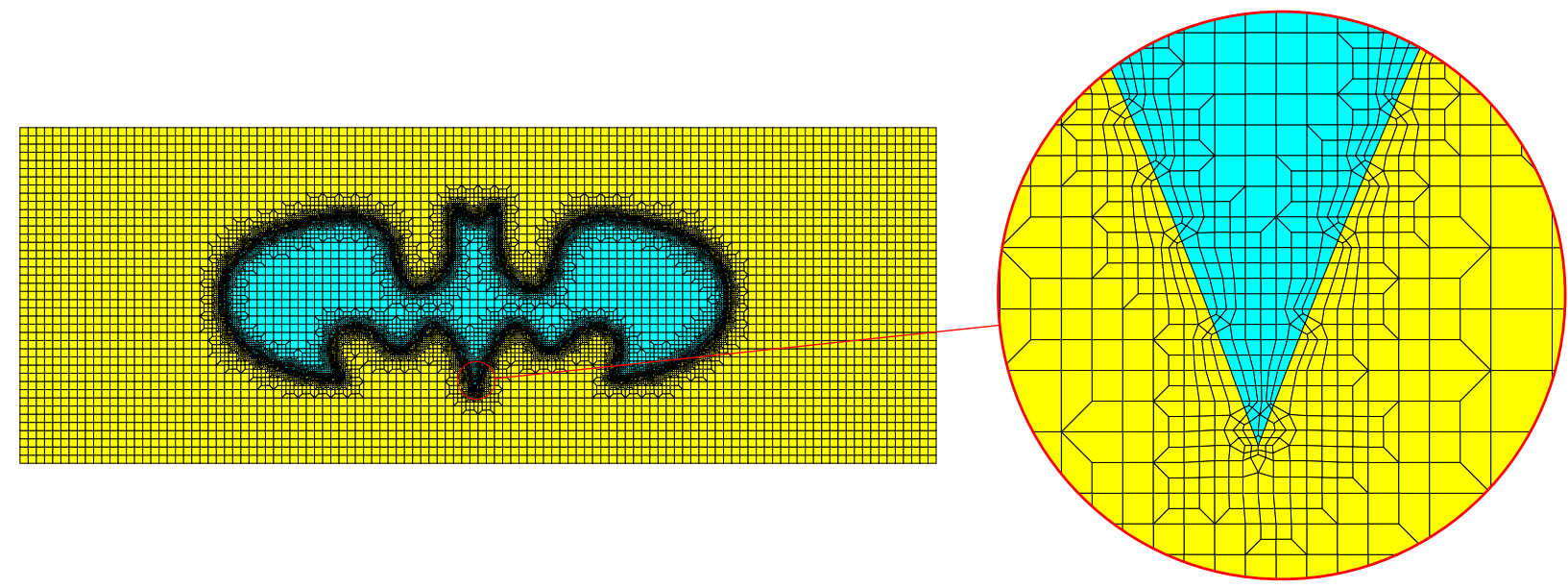

(b) Final all-quad mesh and a magnified region.

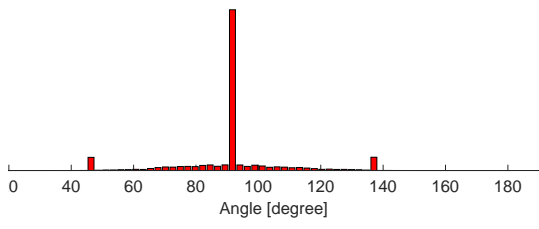

(c) Angle histogram.

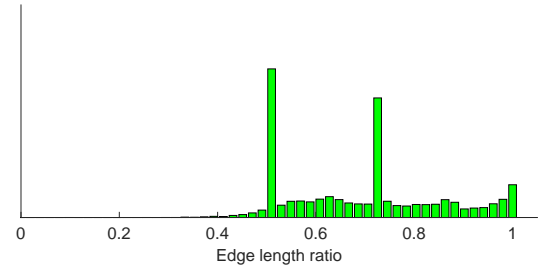

(d) Edge length histogram.

Figure 20: Application of the all-quad meshing algorithm to batman-shaped domain with sharp corners. 


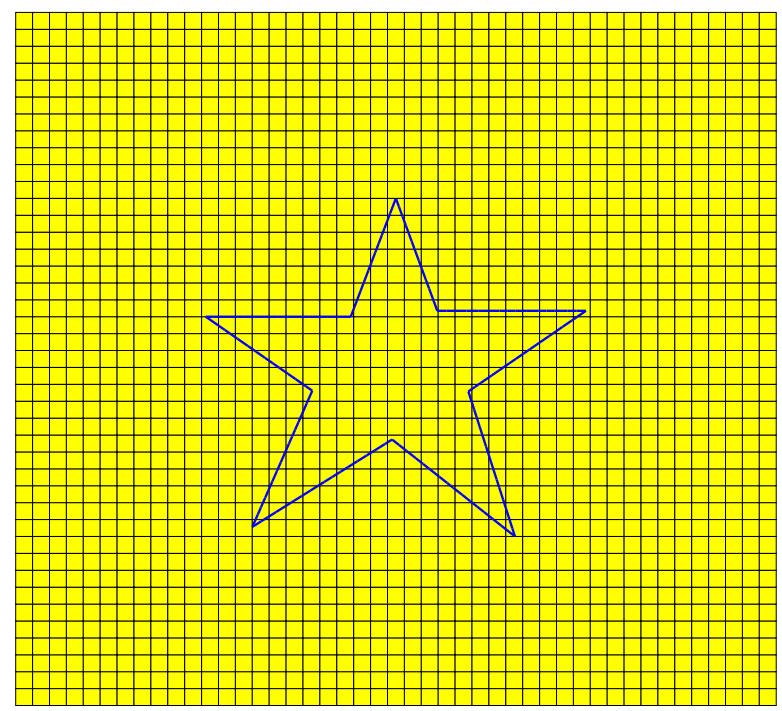

(a) Initial grid and domain.
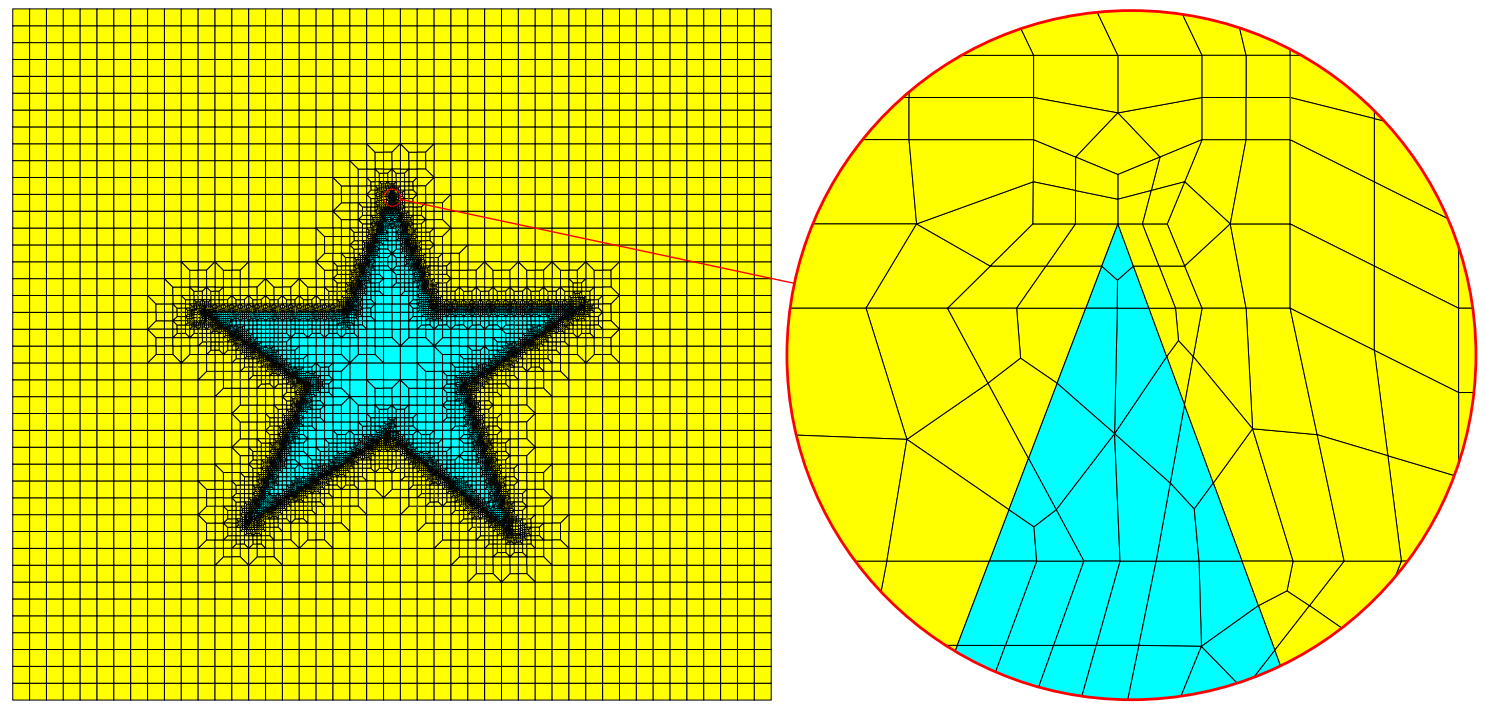

(b) Final all-quad mesh and a magnified region.

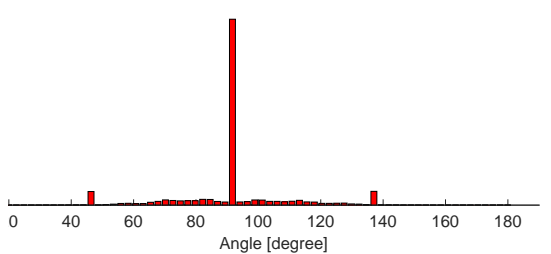

(c) Angle histogram.

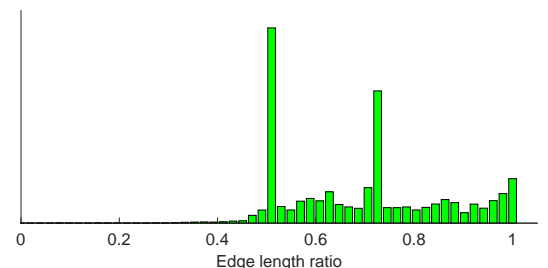

(d) Edge length histogram.

Figure 21: Application of the all-quad meshing algorithm to star-shaped domains with sharp corners. 


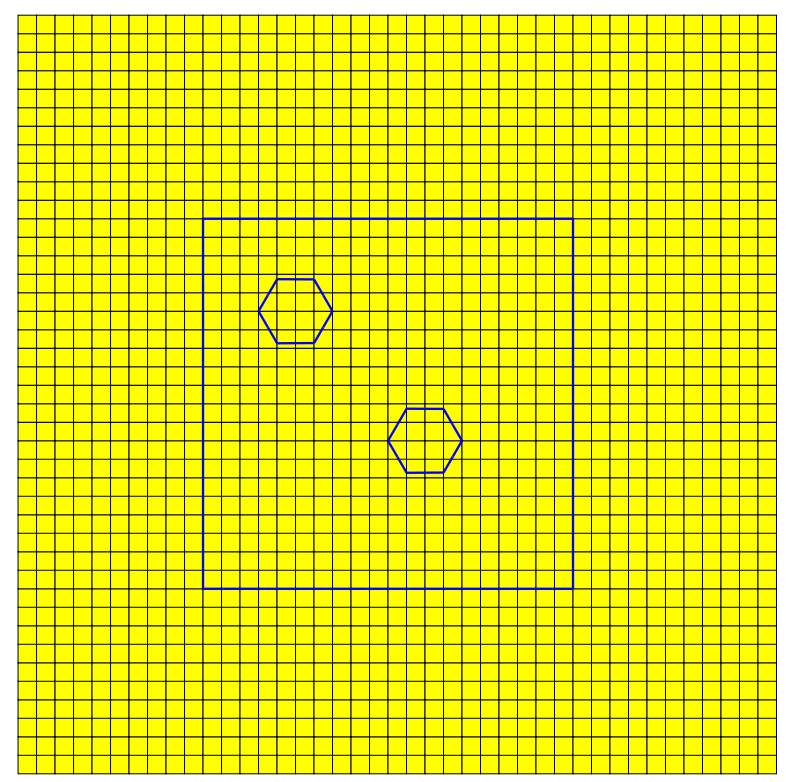

(a) Initial grid and domain.

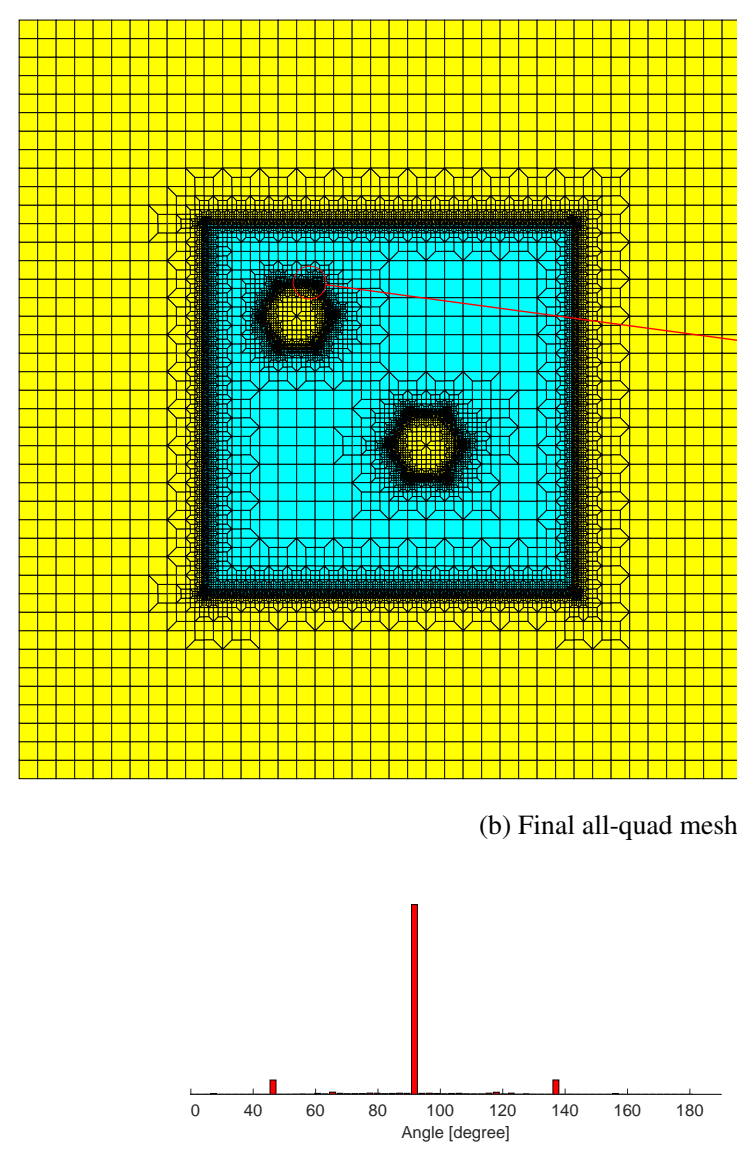

(c) Angle histogram.

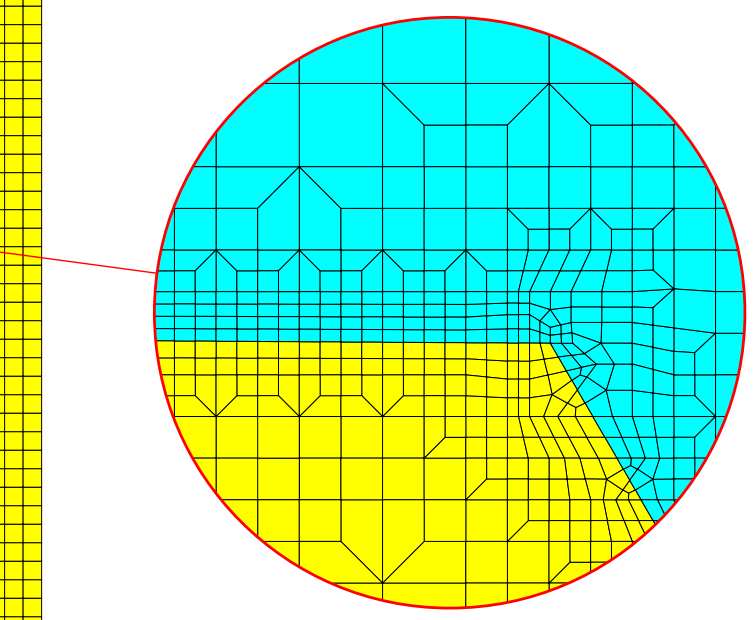

a magnified region.

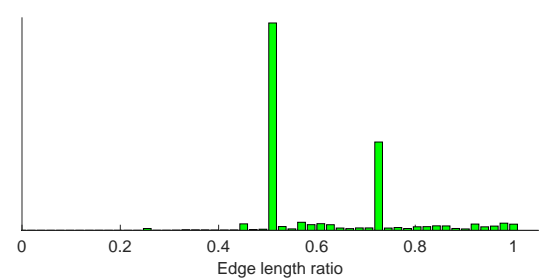

(d) Edge length histogram.

Figure 22: Application of the all-quad meshing algorithm to a double-hex domain with sharp corners. 


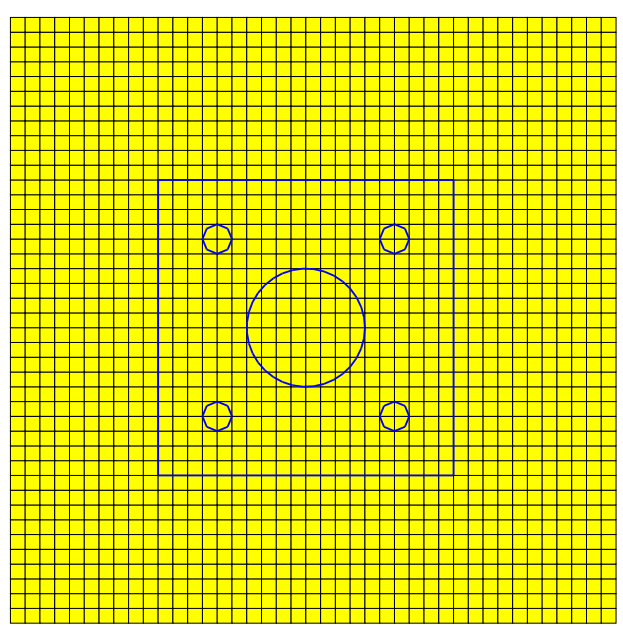

(a) Initial grid and domain.
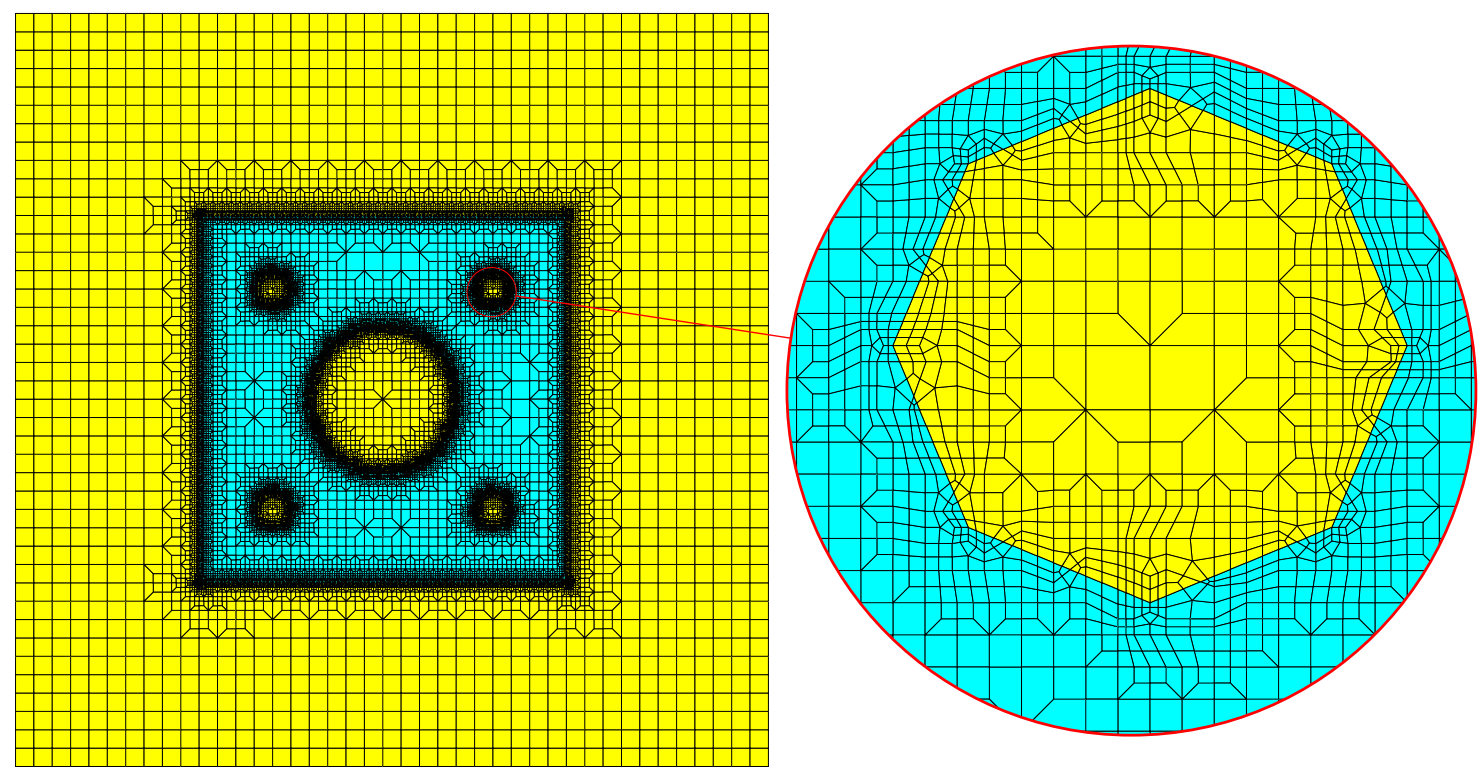

(b) Final all-quad mesh and a magnified region.

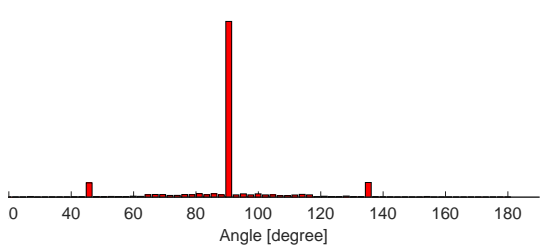

(c) Angle histogram.

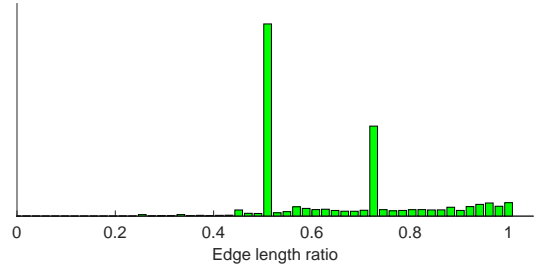

(d) Edge length histogram.

Figure 23: Application of the all-quad meshing algorithm to a domain with sharp corners: square with five eight-sided holes. 